Antonio Ambrosetti · Veronica Felli · Andrea Malchiodi

\title{
Ground states of nonlinear Schrödinger equations with potentials vanishing at infinity
}

Received March 4, 2004

Abstract. We deal with a class on nonlinear Schrödinger equations (NLS) with potentials $V(x) \sim$ $|x|^{-\alpha}, 0<\alpha<2$, and $K(x) \sim|x|^{-\beta}, \beta>0$. Working in weighted Sobolev spaces, the existence of ground states $v_{\varepsilon}$ belonging to $W^{1,2}\left(\mathbb{R}^{N}\right)$ is proved under the assumption that $\sigma<p<$ $(N+2) /(N-2)$ for some $\sigma=\sigma_{N, \alpha, \beta}$. Furthermore, it is shown that $v_{\varepsilon}$ are spikes concentrating at a minimum point of $\mathcal{A}=V^{\theta} K^{-2 /(p-1)}$, where $\theta=(p+1) /(p-1)-1 / 2$.

Keywords. Nonlinear Schrödinger equations, weighted Sobolev spaces

\section{Introduction}

This paper deals with existence of ground state solutions of stationary nonlinear Schrödinger equations of the form

$$
\begin{cases}-\varepsilon^{2} \Delta v+V(x) v=K(x) v^{p}, & x \in \mathbb{R}^{N}, \\ v \in W^{1,2}\left(\mathbb{R}^{N}\right), \quad v(x)>0, & \lim _{x \mid \rightarrow \infty} v(x)=0 .\end{cases}
$$

Here and below, $N \geq 3$ and $1<p<(N+2) /(N-2)$. A solution of (NLS) is called a ground state if it is a Mountain-Pass critical point of the corresponding Euler functional, and hence its Morse index is 1 . If $u$ is a solution of (NLS), then

$$
\psi(x, t)=\exp \left(i \lambda \varepsilon^{-1} t\right) u(x)
$$

represents a standing wave of the nonlinear Schrödinger equation

$$
i \varepsilon \frac{\partial \psi}{\partial t}=-\varepsilon^{2} \Delta \psi+(V(x)-\lambda) \psi-K(x)|\psi|^{p-1} \psi,
$$

where $\varepsilon(=\hbar)$ is the Planck constant and $i$ is the imaginary unit.

One of the main purposes of this paper is to look for solutions $v_{\varepsilon}$ of (NLS) which have the following properties:

A. Ambrosetti: SISSA, via Beirut 2-4, 34014 Trieste, Italy; e-mail: ambr@ sissa.it V. Felli: Univ. Milano Bicocca, Dip. Mat. e Appl., 20126 Milano, Italy; e-mail: felli@matapp.unimib.it

A. Malchiodi: SISSA, via Beirut 2-4, 34014 Trieste, Italy; e-mail: malchiod@ sissa.it 
(i) $v_{\varepsilon} \in W^{1,2}\left(\mathbb{R}^{N}\right)$;

(ii) $v_{\varepsilon}$ is a ground state.

As for (i), let us point out that standing waves $v$ which have finite $L^{2}$ norm are the most relevant from the physical point of view since they correspond to bound states. Moreover, if $v \in W^{1,2}\left(\mathbb{R}^{N}\right)$, one can prove that $\lim _{|x| \rightarrow \infty} v(x)=0$ (see the proof of Theorem 16), which implies that solutions are well localized in space.

On the other hand, concerning (ii), the interest in searching ground states relies on the fact that a standing wave is possibly orbitally stable provided it corresponds to a ground state of (NLS], in the sense specified in the literature (see e.g. [14, 17]).

A lot of work has been devoted to the existence of solutions of (NLS), both for $\varepsilon=1$ and for $\varepsilon$ tending to zero. In the latter case, as a specific feature of the nonlinear (focusing) model, solutions concentrate at points with a soliton profile. We limit ourselves to citing a few recent papers $[5,6,8,9,11,12,15,16]$, referring to their bibliography for a broader list of works, although still not exhaustive.

However, to our knowledge, it is everywhere assumed (with the only exception of [18, 20]) that $\liminf _{|x| \rightarrow \infty} V(x)>0$. The main new feature of the present paper is that we will be concerned with potentials $V$ such that $\lim _{|x| \rightarrow \infty} V=0$.

Our main results are Theorems 1 and 3 . The former deals with existence of ground states of (NLS), the latter with concentration.

Roughly, (NLS) has a ground state which concentrates at a global minimum point of the auxiliary potential $\mathcal{A}:=V^{\theta} K^{-2 /(p-1)}$, where $\theta=(p+1) /(p-1)-N / 2$, provided

(i) $V(x) \sim|x|^{-\alpha}$ with $0<\alpha<2$,

(ii) $K(x) \sim|x|^{-\beta}$ with $\beta>0$,

(iii) $\sigma<p<(N+2) /(N-2)$, where $\sigma$ is a number depending upon $\alpha$ and $\beta$, and defined in (2) below.

Some comments on the proof and the preceding assumptions are in order. If we deal with a potential $V$ which decays to zero at infinity, the methods used in the preceding papers cannot be employed. First of all, variational theory in $W^{1,2}\left(\mathbb{R}^{N}\right)$, as in [11, 12], cannot be used. Nor can one apply perturbation methods, as in [6, 16], since the spectrum of the linear operator $-\Delta+V$ is $[0, \infty)$ (see [10]).

To overcome this difficulty, we frame our problem in a class of weighted Sobolev spaces $\mathcal{H}_{\varepsilon}$, discussed in [19], consisting of the functions $u$ on $\mathbb{R}^{N}$ for which

$$
\int_{\mathbb{R}^{N}}\left(\varepsilon^{2}|\nabla u(x)|^{2}+V(x) u^{2}(x)\right) d x<\infty .
$$

In these spaces the nonlinear term $\int_{\mathbb{R}^{N}} K|u|^{p+1} d x$ is well defined if (i)-(iii) hold. Moreover, under these conditions the Euler functional satisfies the Palais-Smale compactness condition on $\mathcal{H}_{\varepsilon}$, and this allows us to find in a straightforward way a positive MountainPass solution $v_{\varepsilon} \in \mathcal{H}_{\varepsilon}$ (see Theorem 13). It is worth pointing out that for such a result it suffices to assume that (i) holds with $0<\alpha \leq 2$.

However, we are interested in solutions which belong to $W^{1,2}$ and which decay to zero at infinity. To achieve these conditions we first prove some careful integral estimates 
for solutions in $\mathcal{H}_{\varepsilon}$. The proof of the concentration phenomenon also relies on some sharp pointwise decay estimates and on appropriate bounds of the energy of the Mountain-Pass solutions $v_{\varepsilon}$, uniformly with respect to $\varepsilon$. These estimates require $\alpha$ to be smaller than 2 and represent one of the main novelties of the present paper.

As for the assumptions, we point out that if $V(x) \sim|x|^{-\alpha}$ with $0<\alpha \leq 2$, then (iii) cannot be eliminated if we want to find ground states. For more details concerning this claim, we refer to Proposition 15 in Section 4. Concerning assumption (ii), see also Remark14(i).

As already pointed out, the only papers dealing with equations on $\mathbb{R}^{N}$ with potentials vanishing at infinity are [18] and [20]. The former deals with an eigenvalue problem in the radial case. In the latter, weighted Sobolev spaces have also been used. For more details, see Remark 14(ii)-(iii) later on. However, in both the aforementioned papers neither results concerning the fact that the solutions belong to $W^{1,2}\left(\mathbb{R}^{N}\right)$ are given, nor concentration is proved.

The rest of the paper is organized as follows. Section 2 contains our assumptions and main results. Section 3 is devoted to discussing the weighted spaces $\mathcal{H}_{\varepsilon}$ (including an embedding theorem from [19]), as well as to proving some uniform integral estimates that are used in what follows. In Section 4 we deal with the main existence result, Theorem 1 We first prove (see Theorem 13) that in $\mathcal{H}_{\varepsilon}$ the Mountain-Pass Theorem applies in a direct way for any $0<\alpha \leq 2$; next, we assume that $0<\alpha<2$ and prove some exponential decay for the above Mountain-Pass critical points, which allows us to show that they give rise to ground states of (NLS); see Theorem 16 . Finally, in Section 5 we prove that these ground states are spikes concentrating at a minimum point of $\mathcal{A}$. This result is achieved by using the preceding decay estimates, jointly with a uniform bound on the energy of the Mountain-Pass critical points found before.

Notation. Throughout the paper we will use the following notation:

- $B_{R}$ is the ball $\left\{x \in \mathbb{R}^{N}:|x|<R\right\}$;

- $W^{k, p}(\Omega), W^{k, p}\left(\mathbb{R}^{N}\right)$ are the usual Sobolev spaces;

- $L^{p}(\Omega), L^{p}\left(\mathbb{R}^{N}\right)$ are the usual Lebesgue spaces;

- $c, c_{1}, \ldots, C, C_{1}, \ldots$ denote possibly different constants;

- $h_{1} \sim h_{2}$ means that $h_{1}$ and $h_{2}$ are of the same order as $\varepsilon \rightarrow 0$.

\section{Assumptions and main results}

In order to find solutions of (NLS) we will make the following assumptions on $V$ and $K$ :

(V) $\quad V: \mathbb{R}^{N} \rightarrow \mathbb{R}$ is smooth and there exist $\alpha, a, A>0$ such that

$$
\frac{a}{1+|x|^{\alpha}} \leq V(x) \leq A,
$$

( $K) \quad K: \mathbb{R}^{N} \rightarrow \mathbb{R}$ is smooth and there exist $\beta, k>0$ such that

$$
0<K(x) \leq \frac{k}{1+|x|^{\beta}} .
$$


In order to prove existence of ground states of (NLS) as well as their concentration properties we assume a suitable bound on $p$ involving $\alpha$ and $\beta$. Let

$$
\sigma=\sigma_{N, \alpha, \beta}= \begin{cases}\frac{N+2}{N-2}-\frac{4 \beta}{\alpha(N-2)} & \text { if } 0<\beta<\alpha, \\ 1 & \text { otherwise. }\end{cases}
$$

Our main existence result is the following.

Theorem 1. Let $(V),(K)$ hold, with $0<\alpha<2$ and $\beta>0$, respectively, and suppose $p$ satisfies

$$
\sigma<p<\frac{N+2}{N-2}
$$

Then for every $\varepsilon>0$ equation (NLS) has a positive classical solution $v_{\varepsilon} \in W^{1,2}\left(\mathbb{R}^{N}\right)$. Moreover, $v_{\varepsilon}$ is a ground state of the energy functional corresponding to (NLS).

Remark 2. (i) The ground state found above is obtained as Mountain-Pass of the energy functional associated to 19 or, equivalently, it realizes the following supremum:

$$
\sup _{u \in \mathcal{H}_{\varepsilon} \backslash\{0\}} \frac{\int_{\mathbb{R}^{N}} K|u|^{p+1}}{\int_{\mathbb{R}^{N}}\left[\varepsilon^{2}|\nabla u|^{2}+V u^{2}\right]^{(p+1) / 2}},
$$

where $\mathcal{H}_{\varepsilon}$ is a suitable weighted Sobolev space defined in Section 3 Such a supremum is $+\infty$ if $p<\sigma$ as well as if $p>(N+2) /(N-2)$. For more details we refer to Proposition 15

(ii) If $0<\beta<\alpha$, then $\sigma>1$ and the range of $p$ in (3) is smaller than the usual one $1<p<(N+2) /(N-2)$. If $\beta=0$, we would have $\sigma=(N+2) /(N-2)$ and the interval of admissible $p$ would be empty.

(iii) When $\alpha=2$ we can still find a solution in $\mathcal{H}_{\varepsilon}$ but not in $W^{1,2}\left(\mathbb{R}^{N}\right.$ ) (see Theorem 13 .

Concerning semiclassical states of (NLS) we show the following concentration behavior.

Theorem 3. Let the assumptions of Theorem 1 hold. Then the ground state $v_{\varepsilon}$ concentrates at a global minimum point $x^{*}$ of $\mathcal{A}=V^{\theta} K^{-2 /(p-1)}$ with $\theta=(p+1) /(p-1)-N / 2$. More precisely, $v_{\varepsilon}$ has a unique maximum point $x_{\varepsilon}$ with $x_{\varepsilon} \rightarrow x^{*}$ as $\varepsilon \rightarrow 0$, and

$$
v_{\varepsilon}(x)=U^{*}\left(\frac{x-x_{\varepsilon}}{\varepsilon}\right)+\omega_{\varepsilon}(x) \quad \text { as } \varepsilon \rightarrow 0,
$$

where $\omega_{\varepsilon} \rightarrow 0$ in $C_{\mathrm{loc}}^{2}\left(\mathbb{R}^{N}\right)$ and in $L^{\infty}\left(\mathbb{R}^{N}\right)$ as $\varepsilon \rightarrow 0$, and $U^{*}$ is the unique positive radial solution of

$$
-\Delta U^{*}+V\left(x^{*}\right) U^{*}=K\left(x^{*}\right)\left(U^{*}\right)^{p}
$$

The proofs of the above two theorems will be carried out in the rest of the paper. 


\section{Some weighted Sobolev spaces}

As anticipated in the Introduction, we will work in a class of weighted Sobolev spaces. Precisely, let us set, for all $\varepsilon>0$,

$$
\mathcal{H}_{\varepsilon}=\left\{u \in \mathcal{D}^{1,2}\left(\mathbb{R}^{N}\right): \int_{\mathbb{R}^{N}}\left[\varepsilon^{2}|\nabla u(x)|^{2}+V(x) u^{2}(x)\right] d x<\infty\right\} .
$$

$\mathcal{H}_{\varepsilon}$ is a Hilbert space with scalar product and norm, respectively,

$$
\begin{aligned}
\|u\|_{\varepsilon}^{2} & =\int_{\mathbb{R}^{N}}\left[\varepsilon^{2}|\nabla u(x)|^{2}+V(x) u^{2}(x)\right] d x, \\
(u \mid v)_{\varepsilon} & =\int_{\mathbb{R}^{N}}\left[\varepsilon^{2} \nabla u(x) \cdot \nabla v(x)+V(x) u(x) v(x)\right] d x .
\end{aligned}
$$

Set $\mathcal{H}=\mathcal{H}_{1}$ with norm $\|\cdot\|_{\mathcal{H}}$.

Remark 4. Since $V$ is positive and uniformly bounded, it follows that $W^{1,2}\left(\mathbb{R}^{N}\right) \subset \mathcal{H}_{\varepsilon}$ for all $\varepsilon>0$.

Denote by $L_{K}^{q}$ the weighted space of measurable $u: \mathbb{R}^{N} \rightarrow \mathbb{R}$ such that

$$
|u|_{q, K}=\left[\int_{\mathbb{R}^{N}} K(x)|u(x)|^{q} d x\right]^{1 / q}<\infty .
$$

$\mathcal{H}_{\varepsilon}$ and $L_{K}^{q}$ are particular cases of weighted spaces discussed in [19], where the following result is proved.

Theorem 5. Let $N \geq 3$ and suppose that $(V),(K)$ hold with $\alpha \in(0,2]$ and $\beta>0$, respectively. Then for all $\varepsilon>0, \mathcal{H}_{\varepsilon} \subset L_{K}^{p+1}$ provided

$$
\sigma \leq p \leq \frac{N+2}{N-2}
$$

and there is $C_{\varepsilon}>0$ such that

$$
|u|_{q, K} \leq C_{\varepsilon}\|u\|_{\varepsilon}, \quad \forall u \in \mathcal{H}_{\varepsilon} .
$$

Furthermore, the embedding of $\mathcal{H}_{\varepsilon}$ into $L_{K}^{p+1}$ is compact if (3) holds.

In view of this theorem we will assume in what follows that $p, \alpha$ and $\beta$ always satisfy (3).

Remark 6. (i) If $a \leq V(x) \leq A$, that is, when $\alpha=0$, we have $\mathcal{H}_{\varepsilon}=W^{1,2}\left(\mathbb{R}^{N}\right)$ and Theorem 5 implies that $W^{1,2}\left(\mathbb{R}^{N}\right)$ is compactly embedded in $L_{K}^{p+1} \operatorname{provided}(\beta>0$ and) (3) holds.

(ii) If $V(x) \sim\left(1+|x|^{\alpha}\right)^{-1}$ and $K(x) \sim\left(1+|x|^{\beta}\right)^{-1}$, with $0<\alpha \leq 2$ and $\beta>0$, it is proved in [19] that the growth restriction $\sigma \leq p \leq(N+2) /(N-2)$ is a necessary condition for $\mathcal{H}_{\varepsilon}$ to be embedded into $L_{K}^{p+1}\left(\mathbb{R}^{N}\right)$ (see also Proposition 15 . 
In the rest of this section we will prove some integral estimates for functions in $\mathcal{H}_{\varepsilon}$, uniform with respect to $\varepsilon$. We anticipate that, as a byproduct, we will deduce a proof of the embedding result stated in Theorem 5 (see Remark 10 below).

Proposition 7. Let $0<\alpha<2$ and let $p$ satisfy (3). Then for all $\delta>0$ there exists $\bar{R}>0$ such that, for all $R \geq \bar{R}$ and all $u \in \mathcal{H}_{\varepsilon}$ with $\operatorname{supp}(u) \cap B_{R}=\emptyset$, one has

$$
\int_{\mathbb{R}^{N}} K|u|^{p+1} \leq \delta \varepsilon^{-(p-1) N / 2}\|u\|_{\varepsilon}^{p+1}
$$

Proof. The proof is carried out in several steps. First, let us introduce some quantities we need in the proof:

(i) the sequence of radii $R_{n, \varepsilon}$ defined by

$$
R_{n, \varepsilon}=\varepsilon R_{n}, \quad R_{n}=\left[\frac{2-\alpha}{a^{1 / 2}} n\right]^{2 /(2-\alpha)} ;
$$

(ii) the sequence of continuous functions $\psi_{n, \varepsilon}: \mathbb{R}_{+} \rightarrow[0,1]$ satisfying

$$
\psi_{1, \varepsilon}(r)= \begin{cases}1 & \text { if } 0 \leq r \leq R_{n-1, \varepsilon} \\ -\frac{r-R_{1, \varepsilon}}{R_{2, \varepsilon}-R_{1, \varepsilon}}+1 & \text { if } R_{1, \varepsilon} \leq r \leq R_{2, \varepsilon} \\ 0 & \text { if } r \geq R_{2, \varepsilon}\end{cases}
$$

and for $n \geq 2$,

$$
\psi_{n, \varepsilon}(r)= \begin{cases}0 & \text { if } 0 \leq r \leq R_{n-1, \varepsilon} \\ \frac{r-R_{n, \varepsilon}}{R_{n, \varepsilon}-R_{n-1, \varepsilon}}+1 & \text { if } R_{n-1, \varepsilon} \leq r \leq R_{n, \varepsilon} \\ -\frac{r-R_{n, \varepsilon}}{R_{n+1, \varepsilon}-R_{n, \varepsilon}}+1 & \text { if } R_{n, \varepsilon} \leq r \leq R_{n+1, \varepsilon} \\ 0 & \text { if } r \geq R_{n+1, \varepsilon}\end{cases}
$$

(iii) the sequence of sets

$$
A_{n, \varepsilon}=\left\{x \in \mathbb{R}^{N}: R_{n-1, \varepsilon} \leq|x| \leq R_{n+1, \varepsilon}\right\} .
$$

Note that $A_{1, \varepsilon}$ is a ball, $A_{n, \varepsilon}$ is an annulus for $n \geq 2$, and the $\psi_{n, \varepsilon}$ 's have been chosen in such a way that

$$
u(x)=\sum_{n} \psi_{n, \varepsilon}(|x|) u(x)
$$

These cut-off functions are useful to estimate integrals over $\mathbb{R}^{N}$ by means of a discrete sum of integrals on the annuli $A_{n, \varepsilon}$. 
Lemma 8. There exists $c>0$ such that

$$
\int_{\mathbb{R}^{N}} K|u|^{p+1} \leq c \sum_{n=1}^{\infty} \frac{1}{1+R_{n, \varepsilon}^{\beta}} \int_{A_{n, \varepsilon}}\left|\psi_{n, \varepsilon} u\right|^{p+1}, \quad \forall u \in \mathcal{H}_{\varepsilon}, \forall \varepsilon \in(0,1] .
$$

Proof. On $A_{n, \varepsilon}$ one has $u=\psi_{n-1, \varepsilon} u+\psi_{n, \varepsilon} u+\psi_{n+1, \varepsilon} u$ (with abuse of notation we are taking $\left.A_{0, \varepsilon}=\emptyset\right)$, which implies

$$
\int_{A_{n, \varepsilon}} K|u|^{p+1} \leq \sup _{A_{n, \varepsilon}} K\left(\int_{A_{n-1, \varepsilon}}\left|\psi_{n, \varepsilon} u\right|^{p+1}+\int_{A_{n, \varepsilon}}\left|\psi_{n, \varepsilon} u\right|^{p+1}+\int_{A_{n+1, \varepsilon}}\left|\psi_{n, \varepsilon} u\right|^{p+1}\right) .
$$

Since the width of $A_{n, \varepsilon}$ is small with respect to $R_{n, \varepsilon}$ there exists $c_{2}=c_{2}(K)$ such that

$$
\sup _{A_{n, \varepsilon}} K \leq c_{2} \frac{1}{1+R_{n+1, \varepsilon}^{\beta}} \leq c_{2} \frac{1}{1+R_{n, \varepsilon}^{\beta}} \leq c_{2} \frac{1}{1+R_{n-1, \varepsilon}^{\beta}} .
$$

The last two formulas imply

$$
\begin{aligned}
\int_{A_{n, \varepsilon}} K|u|^{p+1} \leq & c_{2}\left(\frac{1}{1+R_{n-1, \varepsilon}^{\beta}} \int_{A_{n-1, \varepsilon}}\left|\psi_{n, \varepsilon} u\right|^{p+1}+\frac{1}{1+R_{n, \varepsilon}^{\beta}} \int_{A_{n, \varepsilon}}\left|\psi_{n, \varepsilon} u\right|^{p+1}\right. \\
& \left.+\frac{1}{1+R_{n+1, \varepsilon}^{\beta}} \int_{A_{n+1, \varepsilon}}\left|\psi_{n, \varepsilon} u\right|^{p+1}\right)
\end{aligned}
$$

Summing over all integers $n$ completes the proof.

Next, we estimate each term $\int_{A_{n, \varepsilon}}\left|\psi_{n, \varepsilon} u\right|^{p+1}$. Let $\gamma$ satisfy $\gamma\left(2^{*}-2\right)=p-1$, or equivalently $2^{*} \gamma=(p-1) N / 2$. Then

$$
\int_{A_{n, \varepsilon}}\left|\psi_{n, \varepsilon} u\right|^{p+1}=\int_{A_{n, \varepsilon}}\left|\psi_{n, \varepsilon} u\right|^{2^{*} \gamma+2-2 \gamma} .
$$

Using the Hölder inequality we find that

$$
\int_{A_{n, \varepsilon}}\left|\psi_{n, \varepsilon} u\right|^{p+1} \leq c_{1}\left[\int_{A_{n, \varepsilon}}\left|\psi_{n, \varepsilon} u\right|^{2^{*}}\right]^{\gamma}\left[\int_{A_{n, \varepsilon}}\left|\psi_{n, \varepsilon} u\right|^{2}\right]^{1-\gamma} .
$$

From the embedding of $\mathcal{D}^{1,2}$ into $L^{2^{*}}$ we infer that

$$
\int_{A_{n, \varepsilon}}\left|\psi_{n, \varepsilon} u\right|^{p+1} \leq c_{2}\left[\int_{A_{n, \varepsilon}}\left|\nabla\left(\psi_{n, \varepsilon} u\right)\right|^{2}\right]^{2^{*} \gamma / 2}\left[\int_{A_{n, \varepsilon}}\left|\psi_{n, \varepsilon} u\right|^{2}\right]^{1-\gamma} .
$$

From (6) and (7) we get

$$
\int_{A_{n, \varepsilon}} K|u|^{p+1} \leq c_{3} \frac{1}{1+R_{n, \varepsilon}^{\beta}}\left[\int_{A_{n, \varepsilon}}\left|\nabla\left(\psi_{n, \varepsilon} u\right)\right|^{2}\right]^{2^{*} \gamma / 2}\left[\int_{A_{n, \varepsilon}}\left|\psi_{n, \varepsilon} u\right|^{2}\right]^{1-\gamma} .
$$

We now show 
Lemma 9. We have

$$
\int_{A_{n, \varepsilon}}\left|\nabla\left(\psi_{n, \varepsilon} u\right)\right|^{2} \leq c_{4} \int_{A_{n, \varepsilon}}\left[|\nabla u|^{2}+\varepsilon^{-2} V u^{2}\right] .
$$

Proof. First we estimate

$$
\left|\nabla\left(\psi_{n, \varepsilon} u\right)\right|^{2}=\left|u \nabla \psi_{n, \varepsilon}+\psi_{n, \varepsilon} \nabla u\right|^{2} \leq 2 u^{2}\left|\nabla \psi_{n, \varepsilon}\right|^{2}+2|\nabla u|^{2} .
$$

From the definition of $R_{n, \varepsilon}$ we get

$$
\left|R_{n+1, \varepsilon}-R_{n, \varepsilon}\right|^{2}=\varepsilon^{2}\left|R_{n+1}-R_{n}\right|^{2} \geq c \varepsilon^{2} R_{n+1, \varepsilon}^{\alpha} .
$$

As above, $R_{n+1, \varepsilon}^{-\alpha} \leq c_{5} \inf _{A_{n, \varepsilon}} V$, and we deduce that

$$
\left|\nabla \psi_{n, \varepsilon}\right|^{2} \leq c_{6}\left|R_{n+1, \varepsilon}-R_{n, \varepsilon}\right|^{-2} \leq c_{7} \varepsilon^{-2} V, \quad x \in A_{n, \varepsilon} .
$$

Substituting in (9) and integrating over $A_{n, \varepsilon}$ proves the lemma.

Proof of Proposition 7 completed. Lemma 9 together with (8) yields

$$
\int_{A_{n, \varepsilon}} K|u|^{p+1} \leq c \frac{1}{1+R_{n, \varepsilon}^{\beta}}\left[\int_{A_{n, \varepsilon}}\left[|\nabla u|^{2}+\varepsilon^{-2} V u^{2}\right]\right]^{2^{*} \gamma / 2}\left[\int_{A_{n, \varepsilon}}\left|\psi_{n, \varepsilon} u\right|^{2}\right]^{1-\gamma} .
$$

Let $M, s>0$ and let $\theta, \theta^{\prime}$ be any pair of conjugate exponents ( $M, s, \theta, \theta^{\prime}$ will be fixed appropriately later). For brevity, set

$$
S=S_{n, \varepsilon}=\int_{A_{n, \varepsilon}}\left[|\nabla u|^{2}+\varepsilon^{-2} V u^{2}\right], \quad T=T_{n, \varepsilon}=\int_{A_{n, \varepsilon}}\left|\psi_{n, \varepsilon} u\right|^{2}
$$

so that 10 becomes

$$
\int_{A_{n, \varepsilon}} K|u|^{p+1} \leq c \frac{1}{1+R_{n, \varepsilon}^{\beta}} S^{2^{*} \gamma / 2} \cdot T^{1-\gamma}
$$

Since

$$
\begin{aligned}
S^{2^{*} \gamma / 2} \cdot \frac{T^{1-\gamma}}{1+R_{n, \varepsilon}^{\beta}} & =M \varepsilon^{s} S^{2^{*} \gamma / 2} \cdot M^{-1} \varepsilon^{-s} \frac{T^{1-\gamma}}{1+R_{n, \varepsilon}^{\beta}} \\
& \leq \frac{1}{\theta} M^{\theta} \varepsilon^{s \theta} S^{2^{*} \gamma \theta / 2}+\frac{1}{\theta^{\prime}} M^{-\theta^{\prime}} \varepsilon^{-s \theta^{\prime}} \frac{T^{(1-\gamma) \theta^{\prime}}}{\left(1+R_{n, \varepsilon}^{\beta}\right)^{\theta^{\prime}}}
\end{aligned}
$$

we get

$$
\int_{A_{n, \varepsilon}} K|u|^{p+1} \leq c_{1} M^{\theta} \varepsilon^{s \theta} S^{2^{*} \gamma \theta / 2}+c_{2} M^{-\theta^{\prime}} \varepsilon^{-s \theta^{\prime}} \frac{T^{(1-\gamma) \theta^{\prime}}}{\left(1+R_{n, \varepsilon}^{\beta}\right)^{\theta^{\prime}}} .
$$

Now we choose $s, \theta$ satisfying

$$
2^{*} \gamma \theta=p+1, \quad \theta\left(s-2^{*} \gamma\right)=-s \theta^{\prime} .
$$


Then

$$
S^{2^{*} \gamma \theta / 2}=\left[\int_{A_{n, \varepsilon}}\left[|\nabla u|^{2}+\varepsilon^{-2} V u^{2}\right]\right]^{2^{*} \gamma \theta / 2}=\varepsilon^{-(p+1)}\left[\int_{A_{n, \varepsilon}}\left[\varepsilon^{2}|\nabla u|^{2}+V u^{2}\right]\right]^{(p+1) / 2},
$$

and hence

$$
M^{\theta} \varepsilon^{s \theta} S^{2^{*} \gamma \theta / 2}=M^{\theta} \varepsilon^{-(p-1) N / 2}\left[\int_{A_{n, \varepsilon}}\left[\varepsilon^{2}|\nabla u|^{2}+V u^{2}\right]\right]^{(p+1) / 2} .
$$

On the other hand, we also have

$$
\begin{aligned}
\frac{T^{(1-\gamma) \theta^{\prime}}}{\left(1+R_{n, \varepsilon}^{\beta}\right)^{\theta^{\prime}}} & \leq \frac{1}{\left(1+R_{n, \varepsilon}^{\beta}\right)^{\theta^{\prime}}}\left[\int_{A_{n, \varepsilon}} u^{2}\right]^{(p+1) / 2} \\
& =\frac{\left(1+R_{n, \varepsilon}^{\alpha}\right)^{(p+1) / 2}}{\left(1+R_{n, \varepsilon}^{\beta} \theta^{\theta^{\prime}}\right.}\left[\int_{A_{n, \varepsilon}} \frac{u^{2}}{1+R_{n, \varepsilon}^{\alpha}}\right]^{(p+1) / 2} \\
& \leq \frac{\left(1+R_{n, \varepsilon}^{\alpha}\right)^{(p+1) / 2}}{\left(1+R_{n, \varepsilon}^{\beta}\right)^{\theta^{\prime}}}\left[\int_{A_{n, \varepsilon}} V u^{2}\right]^{(p+1) / 2} .
\end{aligned}
$$

Inserting the above inequality and (12) into [11], and taking into account that $-s \theta^{\prime}=$ $\theta\left(s-2^{*} \gamma\right)=-(p-1) N / 2$, we infer that

$$
\begin{aligned}
& \int_{A_{n, \varepsilon}} K|u|^{p+1} \leq c_{3} \varepsilon^{-(p-1) N / 2} \\
& \times\left(M^{\theta}\left[\int_{A_{n, \varepsilon}}\left[\left|\varepsilon^{2} \nabla u\right|^{2}+V u^{2}\right]\right]^{(p+1) / 2}+M^{-\theta^{\prime}} \frac{\left(1+R_{n, \varepsilon}^{\alpha}\right)^{(p+1) / 2}}{\left(1+R_{n, \varepsilon}^{\beta}\right)^{\theta^{\prime}}} \cdot\left[\int_{A_{n, \varepsilon}} V u^{2}\right]^{(p+1) / 2}\right) .
\end{aligned}
$$

Now, let us remark that

$$
\frac{\left(1+R_{n, \varepsilon}^{\alpha}\right)^{(p+1) / 2}}{\left(1+R_{n, \varepsilon}^{\beta}\right)^{\theta^{\prime}}} \sim R_{n, \varepsilon}^{-\beta \theta^{\prime}+\alpha(p+1) / 2} \rightarrow 0 \quad\left(R_{n, \varepsilon} \rightarrow \infty\right),
$$

since $p>\sigma$ implies that $-\beta \theta^{\prime}+\alpha(p+1) / 2<0$. Then, given $\delta>0$, we can choose $M, \bar{R}>0$ such that

$$
M^{\theta}<\frac{\delta}{2 c_{3}}, \quad \text { and } \quad M^{-\theta^{\prime}} R^{-\beta \theta^{\prime}+\alpha(p+1) / 2}<\frac{\delta}{2 c_{3}} \quad \text { for } R \geq \bar{R},
$$

yielding

$$
\int_{A_{n, \varepsilon}} K|u|^{p+1} \leq \delta \varepsilon^{-(p-1) N / 2}\left[\int_{A_{n, \varepsilon}}\left[\varepsilon^{2}|\nabla u|^{2}+V u^{2}\right]\right]^{(p+1) / 2}
$$


provided that $R_{n-1, \varepsilon}>\bar{R}$. Summing over these annuli $A_{n, \varepsilon}$ and using the fact that $\operatorname{supp}(u) \cap B_{R}=\emptyset$ for all $R \geq \bar{R}$ we get

$$
\int_{|x|>R} K|u|^{p+1} \leq \delta \varepsilon^{-(p-1) N / 2} \sum_{n}\left[\int_{A_{n, \varepsilon}}\left[\varepsilon^{2}|\nabla u|^{2}+V u^{2}\right]\right]^{(p+1) / 2} .
$$

Setting

$$
a_{n}=a_{n, \varepsilon}=\int_{A_{n, \varepsilon}}\left[\varepsilon^{2}|\nabla u|^{2}+V u^{2}\right],
$$

one has $\sum a_{n}<\|u\|_{\varepsilon}^{2}<\infty$. Letting $\widetilde{\alpha}_{n}=a_{n} / \sum a_{n}$, we have $0<\widetilde{\alpha}_{n} \leq 1$ for all $n$ and hence $\widetilde{\alpha}_{n}^{(p+1) / 2} \leq \widetilde{\alpha}_{n}$, that is, $a_{n}^{(p+1) / 2} \leq\left(\sum a_{n}\right)^{(p+1) / 2-1} a_{n}$. Summing over all $n$, it follows that $\sum a_{n}^{(p+1) / 2} \leq\left(\sum a_{n}\right)^{(p+1) / 2}<\infty$. This implies

$$
\begin{aligned}
\int_{|x|>R} K|u|^{p+1} & \leq \delta \varepsilon^{-(p-1) N / 2}\left[\sum \int_{A_{n, \varepsilon}}\left[\varepsilon^{2}|\nabla u|^{2}+V u^{2}\right]\right]^{(p+1) / 2} \\
& \leq \delta \varepsilon^{-(p-1) N / 2}\|u\|_{\varepsilon}^{p+1},
\end{aligned}
$$

completing the proof of Proposition7

Remark 10. For $\varepsilon=1$ the preceding arguments give an alternative proof of the embedding result stated in Theorem 5. To see this, let us write $u=\chi_{R} u+\left(1-\chi_{R}\right) u$, where $\chi_{R}$ is a cut-off function such that $\chi_{R} \equiv 0$ on $B_{R}, \chi_{R} \equiv 1$ for $|x| \geq R+1$, and $\chi_{R}$ is linear on $R<|x|<R+1$. For $\sigma<p<(N+2) /(N-2)$ we can use inequality (5) to estimate $\int_{\mathbb{R}^{N}} K\left|\chi_{R} u\right|^{p+1}$, while the integral $\int_{|x| \leq R+1} K\left|\left(1-\chi_{R}\right) u\right|^{p+1}$ can be bounded by using the standard Sobolev embedding theorem. If $\sigma \leq p \leq(N+2) / N-2)$, the above method shows that there exist $C>0$ and $R \gg 1$ for which

$$
\int_{|x|>R} K|u|^{p+1} \leq C\|u\|_{\mathcal{H}}^{(p+1) / 2}, \quad \forall u \in \mathcal{H}
$$

Moreover, modifying the definition of $R_{n, \varepsilon}$ (with a logarithmic dependence on $n$ ) we could also recover the embedding in the case $\alpha=2$.

Proposition 11. Let $0<\alpha<2$ and let $p$ satisfy 3). Then for all $\delta>0$ there exists $\bar{R}>0$ such that for all $R \geq \bar{R}$,

$$
\begin{aligned}
& \int_{|x|>R} K(x)|u(x)|^{p+1} d x \\
& \quad \leq \delta \varepsilon^{-(p-1) N / 2}\left(\int_{|x|>R}\left[\varepsilon^{2}|\nabla u(x)|^{2}+V(x) u^{2}(x)\right] d x\right)^{(p+1) / 2}, \quad \forall u \in \mathcal{H}_{\varepsilon} .
\end{aligned}
$$

Proof. Let $\widetilde{\psi}_{R, \varepsilon}: \mathbb{R}_{+} \rightarrow[0,1]$ be a smooth non-decreasing function such that

$$
\widetilde{\psi}_{R, \varepsilon}(r)= \begin{cases}0 & \text { if } 0 \leq r \leq R-\varepsilon R^{\alpha / 2}, \\ 1 & \text { if } r \geq R\end{cases}
$$


satisfying $\left|\widetilde{\psi}_{R, \varepsilon}^{\prime}(r)\right| \leq 2 \varepsilon^{-1} R^{-\alpha / 2}$. Define, in polar coordinates $(r, \vartheta) \in \mathbb{R}_{+} \times S^{N-1}$,

$$
\tilde{u}_{R, \varepsilon}(r, \vartheta)= \begin{cases}\widetilde{\psi}_{R, \varepsilon}(r) u(2 R-r, \vartheta) & \text { if } R-\varepsilon R^{\alpha / 2} \leq r \leq R, \\ u(r, \vartheta) & \text { if } r>R .\end{cases}
$$

In the annulus $A_{R, \varepsilon}=\left\{R-\varepsilon R^{\alpha / 2}<|x|<R\right\}$ we have (subscripts denote partial derivatives)

$\nabla \widetilde{u}_{R, \varepsilon}=-\widetilde{\psi}_{R, \varepsilon}(r) u_{r}(2 R-r, \vartheta) \mathbf{e}_{r}+\frac{1}{r} \widetilde{\psi}_{R, \varepsilon}(r) u_{\vartheta}(2 R-r, \vartheta) \mathbf{e}_{\vartheta}+\widetilde{\psi}_{R, \varepsilon}^{\prime}(r) u(2 R-r, \vartheta) \mathbf{e}_{r}$, where $\mathbf{e}_{r}=x /|x|$ and $\mathbf{e}_{\vartheta}$ is a unit vector tangent to the unit sphere $\{|x|=1\}$. Thus, in $A_{R, \varepsilon}$ one finds that

$$
\left|\nabla \widetilde{u}_{R, \varepsilon}\right|^{2} \leq c_{1}|\nabla u(2 R-r, \vartheta)|^{2}+c_{2} \varepsilon^{-2} R^{-\alpha} u^{2}(2 R-r, \vartheta) .
$$

Let us explicitly point out that here and below the constants $c_{i}$ do not depend upon $R, \varepsilon$. Integrating in $A_{R, \varepsilon}$ and performing the change of variable $(r, \vartheta) \mapsto(2 R-r, \vartheta)$ we get

$$
\begin{aligned}
\int_{A_{R, \varepsilon}}\left|\nabla \widetilde{u}_{R, \varepsilon}\right|^{2} & \leq c_{3} \int_{R<|x|<R+\varepsilon R^{\alpha / 2}}\left[|\nabla u|^{2}+\varepsilon^{-2} R^{-\alpha} u^{2}\right] \\
& \leq c_{4} \varepsilon^{-2} \int_{R<|x|<R+\varepsilon R^{\alpha / 2}}\left[\varepsilon^{2}|\nabla u|^{2}+V(x) u^{2}\right] .
\end{aligned}
$$

Here we have taken into account that $\widetilde{u}_{R, \varepsilon} \equiv u$ for $|x|>R$. From (14) we infer that

$$
\int_{A_{R, \varepsilon}}\left|\nabla \tilde{u}_{R, \varepsilon}\right|^{2} \leq c_{5} \varepsilon^{-2} \int_{|x|>R}\left[\varepsilon^{2}|\nabla u|^{2}+V(x) u^{2}\right] .
$$

Moreover, similar arguments yield

$$
\int_{A_{R, \varepsilon}} V(x) \widetilde{u}_{R, \varepsilon}^{2} \leq c_{6} \int_{R<|x|<R+\varepsilon R^{\alpha / 2}} V(x) \widetilde{u}_{R, \varepsilon}^{2} \leq c_{6} \int_{|x|>R} V(x) u^{2} .
$$

From (15) and 16] we deduce that

$$
\int_{A_{R, \varepsilon}}\left[\varepsilon^{2}\left|\nabla \widetilde{u}_{R, \varepsilon}\right|^{2}+V(x) \widetilde{u}_{R, \varepsilon}^{2}\right] \leq c_{7} \int_{|x|>R}\left[\varepsilon^{2}|\nabla u|^{2}+V(x) u^{2}\right] .
$$

From the embedding (4) and since $\tilde{u}_{R, \varepsilon}=u$ for $r \geq R$, we get

$$
\int_{|x|>R} K(x)|u|^{p+1} \leq \int_{\mathbb{R}^{N}} K(x)\left|\tilde{u}_{R, \varepsilon}\right|^{p+1} .
$$

From Proposition 7 we have

$$
\begin{aligned}
& \int_{\mathbb{R}^{N}} K\left|\widetilde{u}_{R, \varepsilon}\right|^{p+1} \leq \delta \varepsilon^{-(p-1) N / 2}\left(\int_{\mathbb{R}^{N}}\left[\varepsilon^{2}\left|\nabla \widetilde{u}_{R, \varepsilon}\right|^{2}+V \widetilde{u}_{R, \varepsilon}^{2}\right]\right)^{(p+1) / 2} \\
& \quad \leq \delta \varepsilon^{-(p-1) N / 2}\left(\int_{A_{R, \varepsilon}}\left[\varepsilon^{2}\left|\nabla \widetilde{u}_{R, \varepsilon}\right|^{2}+V \widetilde{u}_{R, \varepsilon}^{2}\right]+\int_{|x|>R}\left[\varepsilon^{2}\left|\nabla \widetilde{u}_{R, \varepsilon}\right|^{2}+V \widetilde{u}_{R, \varepsilon}^{2}\right]\right)^{(p+1) / 2} .
\end{aligned}
$$

From this and (17) we finally find (13). 


\section{Proof of the existence results}

This section is devoted to the proof of Theorem 1 , which is divided into two parts. First, we show the existence of a least-energy solution in $\mathcal{H}_{\varepsilon}$ (see Theorem 13 below); in the second part of the section we prove that such a ground state belongs indeed to $W^{1,2}\left(\mathbb{R}^{N}\right)$.

Let us start by introducing the functional set up. If (3) holds, then Theorem 5 applies, yielding

$$
\int_{\mathbb{R}^{N}} K(x)|u(x)|^{p+1} d x<\infty, \quad \forall u \in \mathcal{H}_{\varepsilon}
$$

Define

$$
\begin{aligned}
I_{\varepsilon}(u) & =\frac{1}{2} \int_{\mathbb{R}^{N}} \varepsilon^{2}|\nabla u(x)|^{2} d x+\frac{1}{2} \int_{\mathbb{R}^{N}} V(x) u^{2}(x) d x-\frac{1}{p+1} \int_{\mathbb{R}^{N}} K(x)|u(x)|^{p+1} d x \\
& =\frac{1}{2}\|u\|_{\varepsilon}^{2}-\frac{1}{p+1} \int_{\mathbb{R}^{N}} K(\varepsilon x)|u(x)|^{p+1} d x .
\end{aligned}
$$

From $(18)$ and $(V)$ it follows that $I_{\varepsilon}$ is well defined on $\mathcal{H}_{\varepsilon}$ for all $\varepsilon>0$. Moreover, $I_{\varepsilon}$ is of class $C^{1}$ and

$$
\begin{aligned}
& \left(I_{\varepsilon}^{\prime}(u) \mid v\right) \\
& =\int_{\mathbb{R}^{N}}\left[\varepsilon^{2} \nabla u(x) \cdot \nabla v(x)+V(x) u(x) v(x)-K(x)|u(x)|^{p-1} u(x) v(x)\right] d x, \quad \forall v \in \mathcal{H}_{\varepsilon} .
\end{aligned}
$$

Hence any critical point $u_{\varepsilon} \in \mathcal{H}_{\varepsilon}$ of $I_{\varepsilon}$ is a weak solution of NLS.

Remark 12. By Remark 6.ii), if $V(x) \sim\left(1+|x|^{\alpha}\right)^{-1}$ and $K(x) \sim\left(1+|x|^{\beta}\right)^{-1}$, with $0<\alpha \leq 2$ and $\beta>0$, then the growth restriction $\sigma \leq p \leq(N+2) /(N-2)$ is necessary in order to work in $\mathcal{H}_{\varepsilon}$ with the functional $I_{\varepsilon}$.

Critical points of $I_{\varepsilon}$ can be found by the Mountain-Pass Theorem in a straightforward way.

Theorem 13. Let $(V),(K)$ hold with $0<\alpha \leq 2, \beta>0$, respectively, and suppose that p satisfies (3). Then

$$
b_{\varepsilon}=\inf _{u \in \mathcal{H}_{\varepsilon} \backslash\{0\}} \max _{t \geq 0} I_{\varepsilon}(t u)
$$

is a critical level of $I_{\varepsilon}$. Hence for all $\varepsilon>0$ the equation

$$
-\varepsilon^{2} \Delta v+V(x) v=K(x) v^{p}, \quad x \in \mathbb{R}^{N},
$$

has a positive (classical) solution $v_{\varepsilon} \in \mathcal{H}_{\varepsilon}$. Moreover, there exists $C>0$ such that

$$
\left\|v_{\varepsilon}\right\|_{\varepsilon}^{2} \leq C b_{\varepsilon} .
$$


Proof. Let $\phi$ be a smooth positive function with compact support in $\mathbb{R}^{N}$. Then (recall that $p>1)$ one has $I_{\varepsilon}(t \phi) \rightarrow-\infty$ as $t \rightarrow+\infty$. Hence $I_{\varepsilon}$ has the M-P geometry. Since $\mathcal{H}_{\varepsilon}$ is compactly embedded into $L_{K}^{p+1}$, standard arguments imply that $b_{\varepsilon}$ is a M-P critical level carrying a critical point $v_{\varepsilon} \in \mathcal{H}_{\varepsilon}$ of $I_{\varepsilon}$ which is a weak solution of (NLS). Since $V$ and $K$ are smooth, local regularity implies that $v_{\varepsilon}$ is in fact a classical solution. It is also standard to see that $v_{\varepsilon}>0$. From

$$
-\varepsilon^{2} \Delta v_{\varepsilon}+V(x) v_{\varepsilon}=K(x) v_{\varepsilon}^{p}
$$

we infer that

$$
\int_{\mathbb{R}^{N}}\left[\varepsilon^{2}\left|\nabla v_{\varepsilon}\right|^{2}+V(x) v_{\varepsilon}^{2}\right] d x=\int_{\mathbb{R}^{N}} K(x) v_{\varepsilon}^{p+1} d x .
$$

Thus

$$
b_{\varepsilon}=I_{\varepsilon}\left(v_{\varepsilon}\right)=\left(\frac{1}{2}-\frac{1}{p+1}\right) \int_{\mathbb{R}^{N}}\left[\varepsilon^{2}\left|\nabla v_{\varepsilon}\right|^{2}+V(x) v_{\varepsilon}^{2}\right] d x=\left(\frac{1}{2}-\frac{1}{p+1}\right)\left\|v_{\varepsilon}\right\|_{\varepsilon}^{2} .
$$

This concludes the proof.

Remark 14. (i) Assumption $(V)$ includes potentials which are bounded away from zero (that is, $0<\inf _{\mathbb{R}^{N}} V \leq \sup _{\mathbb{R}^{N}} V<\infty$ ). In this case, the space $\mathcal{H}_{\varepsilon}$ is nothing but $W^{1,2}\left(\mathbb{R}^{N}\right)$ and in order to recover compactness our approach requires $\beta>0$ (see Remark6 6 i)). Let us recall that when, in addition, also $K$ is bounded away from zero (that is, $0<\inf _{\mathbb{R}^{N}} K \leq \sup _{\mathbb{R}^{N}} K<\infty$ ), proving the existence of solutions to (19) requires appropriate assumptions on $V$ and/or $K$ (see the papers cited in the Introduction and [4]). On the other hand, it is well known that if $\beta=0$ a necessary condition for (NLS) to have a solution is that $\int_{\mathbb{R}^{N}} \partial_{x_{i}} V(x) u^{2}(x) d x=0$. Moreover, if $(V)$ holds with $0<\alpha \leq 2$ and $K$ is bounded away from zero, then the critical level $b_{\varepsilon}$ (or the supremum considered in the statement of Proposition 15p is clearly equal to $\infty$. Of course, it is a different story if we look for solutions that are not ground states. For example, it is proved in [6, 24] that if $0<\inf _{\mathbb{R}^{N}} V \leq \sup _{\mathbb{R}^{N}} V<\infty$ and $0<\inf _{\mathbb{R}^{N}} K \leq \sup _{\mathbb{R}^{N}} K<\infty$, then a solution exists provided $\varepsilon$ is sufficiently small and the auxiliary potential $\mathcal{A}$ has a stable stationary point.

(ii) In [18, Thm. 2] the authors consider the eigenvalue problem

$$
\left\{\begin{array}{l}
-\Delta u+V(|x|) u=\lambda K(|x|) u^{p}, \quad x \in \mathbb{R}^{N}, \\
u \in C_{\operatorname{loc}}^{2}\left(\mathbb{R}^{N}\right), \quad \lim _{|x| \rightarrow \infty} u(x)=0,
\end{array}\right.
$$

proving the existence of positive solutions $\left(\lambda, u_{\lambda}\right), u_{\lambda} \in L^{2^{*}}\left(\mathbb{R}^{N}\right)$ and $u_{\lambda}=$ $O\left(r^{(2-n) / 2}\right), r=|x|$. It is assumed that $V(r) \geq 0$ and $K(r)=O\left(r^{-\beta}\right), \beta>0$. In addition, if $\beta<2$, it is required that $p>(N+2-2 \beta) /(N-2)$. Let us point out that the last condition is stronger than ours $(p>\sigma)$.

(iii) Theorem 13 follows from [20, Thm. 3.1] combined with Theorem 5 . Moreover, the case in which $p=\sigma$ or $p=(N+2) /(N-2)$ is also studied in [20, Thm. 3.2], under some further restrictions on $V$ and $K$. 
It is also worth pointing out that if $\sigma<p$ (here we take $0<\beta<\alpha$, otherwise $\sigma=1$ and $p$ satisfies the usual growth assumption), $I_{\varepsilon}$ has no Mountain-Pass solution.

Proposition 15. If either $p<\sigma$ or $p>(N+2) /(N-2)$, then

$$
\sup _{u \in \mathcal{H}_{\varepsilon} \backslash\{0\}} \frac{\int_{\mathbb{R}^{N}} K|u|^{p+1}}{\left(\int_{\mathbb{R}^{N}}\left[\varepsilon^{2}|\nabla u|^{2}+V u^{2}\right]\right)^{(p+1) / 2}}=\infty .
$$

Proof. We can assume for simplicity that $\varepsilon=1$. Let us consider a function $\Psi$ with compact support, and let

$$
u_{\xi}(x)=\Psi(\lambda(x-\xi)), \quad|\xi| \gg 1, \lambda=|\xi|^{-\alpha / 2} .
$$

From the definition of $u \xi$ and the conditions on $\lambda, \xi$ (see $[21)$, we easily find that

$$
\begin{aligned}
\int|\nabla u \xi|^{2} & =\lambda^{2-N} \int|\nabla \Psi|^{2}, \quad \int V u_{\xi}^{2} \geq \frac{C^{-1}}{|\xi|^{\alpha} \lambda^{N}} \int \Psi^{2}, \\
\int K|u|^{p+1} & \leq \frac{C}{|\xi|^{\beta} \lambda^{N}} \int|\Psi|^{p+1} .
\end{aligned}
$$

Hence it follows that

$\frac{\int_{\mathbb{R}^{N}} K u_{\xi}^{p+1}}{\left(\int_{\mathbb{R}^{N}}\left[\left|\nabla u_{\xi}\right|^{2}+V u_{\xi}^{2}\right]\right)^{(p+1) / 2}} \leq C \frac{|\xi|^{-\beta} \lambda^{-N}}{\left(\lambda^{-N}|\xi|^{-\alpha}\right)^{(p+1) / 2}}=C|\xi|^{\frac{\alpha}{4}[(N+2)-p(N-2)]-\beta} \rightarrow \infty$ as $|\xi| \rightarrow \infty$, because $p<\sigma$.

On the other hand, also in the case $p>(N+2) /(N-2)$ it is standard to see that the above supremum is $\infty$. It is sufficient for example to consider the family of functions $u_{\lambda}(x)=\Psi(\lambda x)$, with $\lambda \rightarrow+\infty$.

In the second part of this section we will show that the Mountain-Pass solutions of NLS found above belong indeed to $W^{1,2}\left(\mathbb{R}^{N}\right)$, provided $0<\alpha<2$.

Theorem 16. Let $(V),(K)$ hold with $0<\alpha<2, \beta>0$, respectively, and suppose that $p$ satisfies (3). Then the Mountain-Pass solution $v_{\varepsilon}$ found in Theorem 13 is a ground state of (NLS). In particular, $v_{\varepsilon} \in W^{1,2}\left(\mathbb{R}^{N}\right), v_{\varepsilon} \in C^{2}\left(\mathbb{R}^{N}\right), v_{\varepsilon}(x)>0$ and $\lim _{|x| \rightarrow \infty} v_{\varepsilon}(x)=0$.

The proof of Theorem 16 requires some preliminary decay estimates, based upon the results discussed in Section 3 . Let us point out that to establish the concentration phenomena discussed in Section 5, the decay is proved with estimates which are uniform in $\varepsilon$.

In these lemmas it is always understood that the assumptions of Theorem 16 hold true.

Lemma 17. Let $v_{\varepsilon}$ be solutions of (19) and suppose there exists $\Gamma>0$ such that

$$
\left\|v_{\varepsilon}\right\|_{\mathcal{H}_{\varepsilon}}^{2} \leq \Gamma \varepsilon^{N}
$$


Then there exists $R_{\Gamma}>0$ such that for all $R \geq R_{\Gamma}$ and all $\Omega_{n, \varepsilon} \subseteq \mathbb{R}^{N} \backslash B_{R}$,

$$
\int_{\Omega_{n+1, \varepsilon}}\left[\varepsilon^{2}\left|\nabla v_{\varepsilon}\right|^{2}+V(x) v_{\varepsilon}^{2}\right] d x \leq \frac{3}{4} \int_{\Omega_{n, \varepsilon}}\left[\varepsilon^{2}\left|\nabla v_{\varepsilon}\right|^{2}+V(x) v_{\varepsilon}^{2}\right] d x,
$$

where $\Omega_{n, \varepsilon}=\mathbb{R}^{N} \backslash B_{R_{n, \varepsilon}}$ and $R_{n, \varepsilon}=\varepsilon n^{2 /(2-\alpha)}$.

Proof. Let $R_{n, \varepsilon}$ be as in the statement, and let $\chi_{n, \varepsilon}(r)$ be piecewise affine functions such that

$$
\chi_{n, \varepsilon}(r) \equiv 0, \quad \forall r \leq R_{n, \varepsilon}, \quad \chi_{n, \varepsilon}(r) \equiv 1, \quad \forall r \geq R_{n+1, \varepsilon} .
$$

By the definition of $R_{n, \varepsilon}$ it follows that

$$
\left|R_{n+1, \varepsilon}-R_{n, \varepsilon}\right| \geq C^{-1} \varepsilon^{(2-\alpha) / 2} R_{n+1, \varepsilon}^{\alpha / 2} \geq C^{-1} \varepsilon R_{n+1, \varepsilon}^{\alpha / 2} .
$$

Then

$$
\varepsilon^{2}\left|R_{n+1, \varepsilon}-R_{n, \varepsilon}\right|^{-2} \leq c_{1} R_{n+1, \varepsilon}^{-\alpha} \leq c_{2} \inf \left\{V(x): R_{n, \varepsilon} \leq|x| \leq R_{n+1, \varepsilon}\right\},
$$

and hence

$$
\varepsilon^{2}\left|\nabla \chi_{n, \varepsilon}(x)\right|^{2} \leq V(x), \quad \forall x \in \mathbb{R}^{N} .
$$

Let us test (19) on $\chi_{n, \varepsilon} v_{\varepsilon}$. Recalling that $\chi_{n, \varepsilon}=0$ on $B_{R_{n, \varepsilon}}$ and that $\chi_{n, \varepsilon} \leq 1$, we get

$$
\begin{aligned}
\int_{\Omega_{n, \varepsilon}} \chi_{n, \varepsilon}\left[\varepsilon^{2}\left|\nabla v_{\varepsilon}\right|^{2}+V v_{\varepsilon}^{2}\right] & =\int_{\Omega_{n, \varepsilon}} \chi_{n, \varepsilon} K v_{\varepsilon}^{p+1}-\varepsilon^{2} \int_{\Omega_{n, \varepsilon}} \nabla v_{\varepsilon} \cdot \nabla \chi_{n, \varepsilon} v_{\varepsilon} \\
& \leq \int_{\Omega_{n, \varepsilon}} K v_{\varepsilon}^{p+1}+\frac{1}{2} \varepsilon^{2} \int_{\Omega_{n, \varepsilon}}\left[\left|\nabla v_{\varepsilon}\right|^{2}+\left|\nabla \chi_{n, \varepsilon}\right|^{2} v_{\varepsilon}^{2}\right] .
\end{aligned}
$$

Using 24] we infer that

$$
\varepsilon^{2} \int_{\Omega_{n, \varepsilon}}\left[\left|\nabla v_{\varepsilon}\right|^{2}+\left|\nabla \chi_{n, \varepsilon}\right|^{2} v_{\varepsilon}^{2}\right] \leq \int_{\Omega_{n, \varepsilon}}\left[\varepsilon^{2}\left|\nabla v_{\varepsilon}\right|^{2}+V(x) v_{\varepsilon}^{2}\right] .
$$

From the last two estimates, it follows that

$$
\begin{aligned}
\int_{\Omega_{n+1, \varepsilon}}\left[\varepsilon^{2}\left|\nabla v_{\varepsilon}\right|^{2}+V v_{\varepsilon}^{2}\right] d x & \leq \int_{\Omega_{n, \varepsilon}} \chi_{n, \varepsilon}\left[\varepsilon^{2}\left|\nabla v_{\varepsilon}\right|^{2}+V v_{\varepsilon}^{2}\right] \\
& \leq \int_{\Omega_{n, \varepsilon}} K v_{\varepsilon}^{p+1}+\frac{1}{2} \int_{\Omega_{n, \varepsilon}}\left[\varepsilon^{2}\left|\nabla v_{\varepsilon}\right|^{2}+V(x) v_{\varepsilon}^{2}\right] .
\end{aligned}
$$

Then, from Proposition 11, if $\delta>0$ is given and $R$ is sufficiently large we deduce that

$$
\begin{aligned}
& \int_{\Omega_{n+1, \varepsilon}}\left[\varepsilon^{2}\left|\nabla v_{\varepsilon}\right|^{2}+V v_{\varepsilon}^{2}\right] d x \\
& \leq \delta \varepsilon^{-(p-1) N / 2}\left(\int_{\Omega_{n, \varepsilon}}\left[\varepsilon^{2}\left|\nabla v_{\varepsilon}\right|^{2}+V(x) v_{\varepsilon}^{2}\right] d x\right)^{(p+1) / 2}+\frac{1}{2} \int_{\Omega_{n, \varepsilon}}\left[\varepsilon^{2}\left|\nabla v_{\varepsilon}\right|^{2}+V(x) v_{\varepsilon}^{2}\right] .
\end{aligned}
$$


Now we write

$$
\begin{aligned}
\left(\int _ { \Omega _ { n , \varepsilon } } \left[\varepsilon^{2}\left|\nabla v_{\varepsilon}\right|^{2}\right.\right. & \left.\left.+V(x) v_{\varepsilon}^{2}\right] d x\right)^{(p+1) / 2} \\
& =\left(\int_{\Omega_{n, \varepsilon}}\left[\varepsilon^{2}\left|\nabla v_{\varepsilon}\right|^{2}+V(x) v_{\varepsilon}^{2}\right] d x\right)^{(p-1) / 2} \int_{\Omega_{n, \varepsilon}}\left[\varepsilon^{2}\left|\nabla v_{\varepsilon}\right|^{2}+V(x) v_{\varepsilon}^{2}\right] d x .
\end{aligned}
$$

From 23 and the last two formulas it follows that

$$
\int_{\Omega_{n+1, \varepsilon}}\left[\varepsilon^{2}\left|\nabla v_{\varepsilon}\right|^{2}+V v_{\varepsilon}^{2}\right] d x \leq\left(\frac{1}{2}+\delta \Gamma^{(p-1) / 2}\right) \int_{\Omega_{n, \varepsilon}}\left[\varepsilon^{2}\left|\nabla v_{\varepsilon}\right|^{2}+V(x) v_{\varepsilon}^{2}\right] d x .
$$

Choosing $\delta$ sufficiently small (and hence for $R$ large) we obtain the assertion.

Lemma 18. Let $v_{\varepsilon}$ be solutions of (19), and let $\Gamma, R_{\Gamma}$ be as above. Then, for all $\rho \geq 2 R_{\Gamma}$,

$$
\begin{aligned}
\int_{|x|>\rho}\left[\varepsilon^{2}\left|\nabla v_{\varepsilon}\right|^{2}+V\right. & \left.(x) v_{\varepsilon}^{2}\right] d x \\
\leq & \bar{C}_{\Gamma} \varepsilon^{N} \exp \left\{-\frac{1}{2}\left|\log \frac{3}{4}\right| \varepsilon^{-1}\left(\rho^{(2-\alpha) / 2}-R_{\Gamma}^{(2-\alpha) / 2}\right)\right\},
\end{aligned}
$$

for some constant $\bar{C}_{\Gamma}$ depending only on $\Gamma$.

Proof. Given $\rho>2 R_{\Gamma}$, let $\tilde{n}>\bar{n}$ be positive integers such that

$$
R_{\bar{n}, \varepsilon} \leq R_{\Gamma} \leq R_{\bar{n}+1, \varepsilon}, \quad R_{\tilde{n}-1, \varepsilon} \leq \rho \leq R_{\tilde{n}, \varepsilon} .
$$

From (17), we deduce that

$$
\begin{aligned}
\int_{|x|>\rho}\left[\varepsilon^{2}\left|\nabla v_{\varepsilon}\right|^{2}+V(x) v_{\varepsilon}^{2}\right] & \leq \int_{|x|>R_{\tilde{n}, \varepsilon}}\left[\varepsilon^{2}\left|\nabla v_{\varepsilon}\right|^{2}+V(x) v_{\varepsilon}^{2}\right] \\
& \leq\left(\frac{3}{4}\right)^{\tilde{n}-\bar{n}} \int_{|x|>R_{\Gamma}}\left[\varepsilon^{2}\left|\nabla v_{\varepsilon}\right|^{2}+V(x) v_{\varepsilon}^{2}\right] .
\end{aligned}
$$

Then 23) implies

$$
\int_{|x|>\rho}\left[\varepsilon^{2}\left|\nabla v_{\varepsilon}\right|^{2}+V(x) v_{\varepsilon}^{2}\right] \leq\left(\frac{3}{4}\right)^{\tilde{n}-\bar{n}} \Gamma \varepsilon^{N} .
$$

By our choices of $\bar{n}, \tilde{n}$,

$$
\rho \sim \varepsilon^{2 /(2-\alpha)} \tilde{n}^{2 /(2-\alpha)}, \quad R_{\Gamma} \sim \varepsilon^{2 /(2-\alpha)} \bar{n}^{2 /(2-\alpha)},
$$

which implies

$$
\tilde{n}-\bar{n} \geq \frac{1}{2} \varepsilon^{-1}\left(\rho^{(2-\alpha) / 2}-R_{\Gamma}^{(2-\alpha) / 2}\right) .
$$

The estimate in 26) and the last formula conclude the proof. 
Proof of Theorem 16 Here $\varepsilon>0$ is fixed and can be taken equal to 1 to simplify the notation. Let $v \in \mathcal{H}$ be any solution of 19 ) (with $\varepsilon=1$ ) and let $y \in \mathbb{R}^{N}$ be such that $|y|>2$. Then

$$
\int_{B_{1}(y)} v^{2}=\int_{B_{1}(y)} V(x) v^{2} \cdot \frac{1}{V(x)} \leq c_{1}|y|^{\alpha} \int_{B_{1}(y)} V(x) v^{2} .
$$

For $R=\frac{1}{2}|y|$ we have

$$
\int_{B_{1}(y)} V(x) v^{2} \leq \int_{\mathbb{R}^{N} \backslash B_{R}} V(x) v^{2} .
$$

From the preceding two estimates and Lemma 18 we get

$$
\int_{B_{1}(y)} v^{2} \leq C_{3}|y|^{\alpha} \exp \left\{-C_{4}|y|^{1-\alpha / 2}\right\}, \quad \forall|y| \gg 1 .
$$

Let $m \in \mathbb{N}$ and $y_{i} \in \mathbb{R}^{N}, i=1, \ldots, m$, be such that $B_{5} \backslash B_{2} \subset \bigcup_{i=1}^{m} B_{1}\left(y_{i}\right)$, and let $y_{i, k}=2^{k} y_{i}$. Then we get

$$
\int_{\mathbb{R}^{N} \backslash B_{2}} v^{2} \leq \sum_{k=0}^{\infty} \int_{2^{k}\left(B_{5} \backslash B_{2}\right)} v^{2} \leq \sum_{i, k} \int_{B_{2^{k}}\left(y_{i, k}\right)} v^{2} .
$$

To estimate the right hand side, we use 27) for $k \gg 1$, which yields

$$
\int_{\mathbb{R}^{N} \backslash B_{2}} v^{2} \leq C_{3} \sum_{i, k}\left|y_{i, k}\right|^{\alpha} \exp \left\{-C_{4}\left|y_{i, k}\right|^{1-\alpha / 2}\right\}<\infty,
$$

since $0<\alpha<2$. This shows that $v \in L^{2}\left(\mathbb{R}^{N}\right)$, whence $v \in W^{1,2}\left(\mathbb{R}^{N}\right)$. As already pointed out in Theorem 13, $v \in C^{2}\left(\mathbb{R}^{N}\right)$ and $v>0$. Finally, standard arguments show that $\lim _{|x| \rightarrow \infty} v(x)=0$ (see for example [22]).

\section{Semiclassical limits for (NLS)}

In this section we study the behavior of some solutions of (NLS) as $\varepsilon$ tends to 0 , and in particular of those obtained in Theorem 13 . We always assume that $(V),(K)$ hold true with $0<\alpha<2$ and $\beta>0$, and that $p$ satisfies (3). However some results, as Lemma 19 below, hold even if $0<\alpha \leq 2$.

The next lemma provides an upper bound for the critical values $b_{\varepsilon}$ in terms of the auxiliary functional $\mathcal{A}=V^{\theta} K^{-2 /(p-1)}$ introduced in Theorem 3 It is worth pointing out explicitly that, since $p>\sigma, \mathcal{A}(x) \rightarrow \infty$ as $|x| \rightarrow \infty$, and therefore $\mathcal{A}$ has a global minimum on all of $\mathbb{R}^{N}$.

Lemma 19. There exists $C_{0}>0$ such that for all $\xi \in \mathbb{R}^{N}$ and all $\varepsilon$ sufficiently small,

$$
\varepsilon^{-N} b_{\varepsilon}=\varepsilon^{-N} I_{\varepsilon}\left(v_{\varepsilon}\right) \leq C_{0} \mathcal{A}(\xi)+o(1) \quad \text { as } \varepsilon \rightarrow 0^{+} .
$$

In particular there exists $C^{*}>0$ such that $b_{\varepsilon} \leq C^{*} \varepsilon^{N}$. 
Proof. For any $\xi \in \mathbb{R}^{N}$, let us define the functional $F_{\xi}$ on $W^{1,2}\left(\mathbb{R}^{N}\right)$ by setting

$$
F_{\xi}(u)=\frac{1}{2} \int_{\mathbb{R}^{N}}|\nabla u|^{2}+\frac{1}{2} V(\xi) \int_{\mathbb{R}^{N}} u^{2}-\frac{1}{p+1} K(\xi) \int_{\mathbb{R}^{N}}|u|^{p+1} .
$$

Let $f(\xi)$ denote the Mountain-Pass critical level of $F_{\xi}$. It is well known that

$$
f(\xi)=\inf _{u \in \mathcal{N}_{\xi}} F_{\xi}(u)
$$

where $\mathcal{N}_{\xi}$ is the Nehari manifold

$$
\mathcal{N}_{\xi}=\left\{u \in W^{1,2}\left(\mathbb{R}^{N}\right) \backslash\{0\}: \int_{\mathbb{R}^{N}}|\nabla u|^{2}+V(\xi) \int_{\mathbb{R}^{N}} u^{2}=K(\xi) \int_{\mathbb{R}^{N}}|u|^{p+1}\right\} .
$$

Let us point out that $u \in \mathcal{N}_{\xi}$ if and only if

$$
\hat{u}(y):=K^{1 /(p-1)}(\xi) V^{-1 /(p-1)}(\xi) u\left(V^{-1 / 2}(\xi) y\right) \in \mathcal{N},
$$

where $\mathcal{N}=\left\{u \in W^{1,2}\left(\mathbb{R}^{N}\right): u \neq 0\right.$ and $\left.\int_{\mathbb{R}^{N}}\left(|\nabla u|^{2}+u^{2}\right)=\int_{\mathbb{R}^{N}}|u|^{p+1}\right\}$. Hence, with a direct calculation we find

$$
\begin{aligned}
f(\xi) & =\inf _{\mathcal{N}_{\xi}} F_{\xi}=\left(\frac{1}{2}-\frac{1}{p+1}\right) K(\xi) \inf _{u \in \mathcal{N}_{\xi}} \int_{\mathbb{R}^{N}}|u|^{p+1} d x \\
& =\left(\frac{1}{2}-\frac{1}{p+1}\right) K^{-2 /(p-1)}(\xi) V^{(p+1) /(p-1)-N / 2}(\xi) \inf _{v \in \mathcal{N}} \int_{\mathbb{R}^{N}}|v|^{p+1} d y .
\end{aligned}
$$

Let $\bar{U}$ denote the unique positive radial solution in $W^{1,2}\left(\mathbb{R}^{N}\right)$ of

$$
-\Delta \bar{U}+\bar{U}=\bar{U}^{p} \quad \text { in } \mathbb{R}^{N} .
$$

Since $\inf _{v \in \mathcal{N}} \int_{\mathbb{R}^{N}}|v|^{p+1} d y$ is achieved at $\bar{U}$, we get

$$
\begin{aligned}
f(\xi) & =[K(\xi)]^{-2 /(p-1)}[V(\xi)]^{(p+1) /(p-1)-N / 2}\left(\frac{1}{2}-\frac{1}{p+1}\right) \int_{\mathbb{R}^{N}}|\bar{U}|^{p+1} d x \\
& =C_{0} \mathcal{A}(\xi) .
\end{aligned}
$$

Since $f(\xi)$ is a Mountain-Pass level of $F_{\xi}$, for all $v>0$ there exists $w \in W^{1,2}\left(\mathbb{R}^{N}\right)$ such that

$$
f(\xi) \leq \max _{t>0} F_{\xi}(t w) \leq f(\xi)+v
$$

Let $\varphi \in C^{2}\left(\mathbb{R}^{N}\right)$ be a cut-off function such that $\varphi \equiv 1$ in a neighborhood of $\xi$ and define, for any $\varepsilon>0, w_{\varepsilon} \in W^{1,2}\left(\mathbb{R}^{N}\right)$ by

$$
w_{\varepsilon}(x)=\varphi(x) w\left(\frac{x-\xi}{\varepsilon}\right) .
$$


Since $W^{1,2}\left(\mathbb{R}^{N}\right) \subset \mathcal{H}_{\varepsilon}$, we have $w_{\varepsilon} \in \mathcal{H}_{\varepsilon}$ for any $\varepsilon$; in particular it makes sense to compute $I_{\varepsilon}\left(t w_{\varepsilon}\right)$, which yields

$$
I_{\varepsilon}\left(t w_{\varepsilon}\right)=\frac{t^{2}}{2}\left\|w_{\varepsilon}\right\|_{\varepsilon}^{2}-\frac{t^{p+1}}{p+1} \int_{\mathbb{R}^{N}} K(x)\left|w_{\varepsilon}\right|^{p+1} d x .
$$

By the change of variable $y=(x-\xi) / \varepsilon$, we get

$$
\begin{aligned}
\varepsilon^{-N}\left\|w_{\varepsilon}\right\|_{\varepsilon}^{2}= & \varepsilon^{2} \int_{\mathbb{R}^{N}}|\nabla \varphi(\varepsilon y+\xi)|^{2} w^{2}(y) d y \\
& +\varepsilon \int_{\mathbb{R}^{N}} \nabla w(y) \cdot \nabla \varphi(\varepsilon y+\xi) w(y) \varphi(\varepsilon y+\xi) d y \\
& +\int_{\mathbb{R}^{N}} \varphi^{2}(\varepsilon y+\xi)\left|\nabla w_{\varepsilon}(y)\right|^{2} d y+\int_{\mathbb{R}^{N}} V(\varepsilon y+\xi) \varphi^{2}(\varepsilon y+\xi) w^{2}(y) d y,
\end{aligned}
$$

as well as

$$
\varepsilon^{-N} \int_{\mathbb{R}^{N}} K(x)\left|w_{\varepsilon}(x)\right|^{p+1} d x=\int_{\mathbb{R}^{N}} K(\varepsilon y+\xi)|\varphi(\varepsilon y+\xi) w(y)|^{p+1} d y .
$$

Putting together the preceding equations we deduce that

$$
\varepsilon^{-N} I_{\varepsilon}\left(t w_{\varepsilon}\right)=F_{\xi}(t w)+o(1) \quad \text { as } \varepsilon \rightarrow 0 .
$$

Hence

$$
\begin{aligned}
\varepsilon^{-N} I_{\varepsilon}\left(v_{\varepsilon}\right) & =\inf _{v \in \mathcal{H}_{\varepsilon} \backslash\{0\}} \max _{t>0} \varepsilon^{-N} I_{\varepsilon}(t v) \\
& \leq \max _{t>0} I_{\varepsilon}\left(t w_{\varepsilon}\right) \leq \max _{t>0} F_{\xi}(t w)+o(1) \\
& \leq f(\xi)+v+o(1)=C_{0} \mathcal{A}(\xi)+v+o(1)
\end{aligned}
$$

Since $v>0$ is arbitrary, the estimate in 28 is proved. The last statement follows from the fact that $\mathcal{A}$ has a global minimum on $\mathbb{R}^{N}$ since $p>\sigma$.

Remark 20. To prove that $b_{\varepsilon} \leq C^{*} \varepsilon^{N}$ one could also argue as follows. Consider the functionals $\widetilde{I}_{\varepsilon}, \widehat{I}_{\varepsilon}: W^{1,2}\left(\mathbb{R}^{N}\right) \rightarrow \mathbb{R}$ defined by

$$
\begin{aligned}
& \widetilde{I}_{\varepsilon}(u)=\frac{1}{2} \int_{\mathbb{R}^{N}}\left[\varepsilon^{2}|\nabla u|^{2}+A u^{2}\right] d x-\frac{1}{p+1} \int_{\mathbb{R}^{N}} K(x)|u|^{p+1} d x, \\
& \widehat{I}_{\varepsilon}(u)=\frac{1}{2} \int_{\mathbb{R}^{N}}\left[|\nabla u|^{2}+A u^{2}\right] d x-\frac{1}{p+1} \int_{\mathbb{R}^{N}} K(\varepsilon x)|u|^{p+1} d x .
\end{aligned}
$$

Clearly, $\widehat{u}_{\varepsilon}(x)$ is a critical point of $\widehat{I}_{\varepsilon}$ iff $\widetilde{u}_{\varepsilon}(x):=\widehat{u}_{\varepsilon}(x / \varepsilon)$ is a critical point of $\widetilde{I}_{\varepsilon}$; moreover, $\widetilde{I}_{\varepsilon}\left(\widetilde{u}_{\varepsilon}\right)=\varepsilon^{N} \widehat{I}_{\varepsilon}\left(\widehat{u}_{\varepsilon}\right)$. Let $\widetilde{b}_{\varepsilon}$, resp. $\widehat{b}_{\varepsilon}$, denote the Mountain-Pass critical level of $\widetilde{I}_{\varepsilon}$, resp. $\widehat{I}_{\varepsilon}$. Since $\sup V \leq A$ and $W^{1,2}\left(\mathbb{R}^{N}\right) \subset \mathcal{H}_{\varepsilon}$, one easily deduces that $b_{\varepsilon} \leq \widetilde{b}_{\varepsilon}=\varepsilon^{N} \widehat{b}_{\varepsilon}$. 
On the other hand, critical points of $\widehat{I}_{\varepsilon}$ can be found near those of the unperturbed functional $\widehat{I}_{0} \equiv \widehat{I}_{\varepsilon=0}$. Up to translation, we can assume that $K(0)=\max K$. Let $U$ be the unique positive radial solution of

$$
-\Delta U+A U=K(0) U^{p}, \quad U \in W^{1,2}\left(\mathbb{R}^{N}\right) .
$$

Then, using [1], one infers that $\widehat{I}_{\varepsilon}$ has, for $\varepsilon>0$ small, a critical point $u_{\varepsilon}$ such that $\widehat{u}_{\varepsilon} \rightarrow U$ as $\varepsilon \rightarrow 0$. In particular, from $\widehat{I}_{\varepsilon}\left(\widehat{u}_{\varepsilon}\right) \rightarrow \widehat{I}_{0}(U)$ it follows that there exists $C>0$ such that $\widehat{I}_{\varepsilon}\left(\widehat{u}_{\varepsilon}\right) \leq C$ for all $\varepsilon>0$ small enough. Moreover, since $U$ is a Mountain-Pass critical point of $\widehat{I}_{0}$, the same holds for $u_{\varepsilon}$. This implies that $\widehat{b}_{\varepsilon} \leq \widehat{I}_{\varepsilon}\left(\widehat{u}_{\varepsilon}\right)$, and the result follows.

Lemma 19 and (20) yield

Corollary 21. For $\varepsilon$ small there exists $\Gamma>0$ such that

$$
\left\|v_{\varepsilon}\right\|_{\varepsilon}^{2} \leq \Gamma \varepsilon^{N}
$$

where $v_{\varepsilon}$ is given by Theorem 13 .

The next lemma provides pointwise uniform decay estimates for the solutions $v_{\varepsilon}$. Here $0<\alpha<2$ is needed. We follow closely the method illustrated in [21, Appendix B].

Lemma 22. Let $\Gamma, R_{\Gamma}$ and $v_{\varepsilon}$ be as in Lemma 17. Then there exists a constant $C$, depending only on $\Gamma, p$ and $N$, and a positive number $d>0$, depending on $N, p, \alpha$ and $\beta$, such that

$$
\left|v_{\varepsilon}(x)\right| \leq C|x|^{d} \varepsilon^{-d} \exp \left\{-\frac{1}{4}\left|\log \frac{3}{4}\right| \varepsilon^{-1}\left(|x|^{(2-\alpha) / 2}-R_{\Gamma}^{(2-\alpha) / 2}\right)\right\} \text { for }|x| \geq 2 R_{\Gamma}+C \text {. }
$$

Proof. The functions $v_{\varepsilon}$ satisfy the equation

$$
-\varepsilon^{2} \Delta v_{\varepsilon}+V(x) v_{\varepsilon}=K(x) v_{\varepsilon}^{p} .
$$

Given $x_{0} \in \mathbb{R}^{N}$ with $\left|x_{0}\right| \geq 2 R_{\Gamma}+2$, we consider a smooth cut-off function $\eta$ satisfying

$$
\eta(x)=\left\{\begin{array}{ll}
1 & \text { for } x \in B_{1}\left(x_{0}\right), \\
0 & \text { for } x \in \mathbb{R}^{N} \backslash B_{2}\left(x_{0}\right),
\end{array} \quad|\nabla \eta| \leq 2 .\right.
$$

Letting for simplicity $v=v_{\varepsilon}$, given $L>0$ and $s \geq 0$, we also define the function $\phi=\phi_{s, L} \equiv v \min \left\{|v|^{2 s}, L^{2}\right\} \eta^{2}$. Testing [31) on $\phi$ we obtain

$$
\begin{aligned}
\varepsilon^{2} \int|\nabla v|^{2} \min \left\{|v|^{2 s},\right. & \left.L^{2}\right\} \eta^{2}+\frac{s}{2} \varepsilon^{2} \int_{\left\{|v|^{s} \leq L\right\}}\left|\nabla\left(|v|^{2}\right)\right|^{2} v^{2 s-2} \eta^{2}+\int V(x) v^{2} \eta^{2} \min \left\{|v|^{2 s}, L^{2}\right\} \\
\leq & -2 \varepsilon^{2} \int v \eta \min \left\{|v|^{2 s}, L^{2}\right\} \nabla v \cdot \nabla \eta+\int K v^{p+1} \eta^{2} \min \left\{|v|^{2 s}, L^{2}\right\} \\
\leq & \frac{1}{2} \varepsilon^{2} \int|\nabla v|^{2} \min \left\{|v|^{2 s}, L^{2}\right\} \eta^{2}+C \varepsilon^{2} \int v^{2} \min \left\{|v|^{2 s}, L^{2}\right\}|\nabla \eta|^{2} \\
& +\int K v^{p+1} \eta^{2} \min \left\{|v|^{2 s}, L^{2}\right\} .
\end{aligned}
$$


Hence, if we set

$$
w=\eta v \min \left\{|v|^{s}, L\right\},
$$

from the above inequality we get

$$
\int \varepsilon^{2}|\nabla w|^{2}+V(x) w^{2} \leq C \varepsilon^{2} \int v^{2} \min \left\{|v|^{2 s}, L^{2}\right\}+\int K v^{p+1} \eta^{2} \min \left\{|v|^{2 s}, L^{2}\right\} .
$$

Next, given $M>0$, we divide the last integral into the two regions $\{v \leq M\}$ and $\{v>M\}$ to obtain

$$
\begin{aligned}
\int K v^{p+1} & \eta^{2} \min \left\{|v|^{2 s}, L^{2}\right\} \\
& \leq M^{p-1} \int K \eta^{2} v^{2} \min \left\{|v|^{2 s}, L^{2}\right\}+\int_{\{v>M\} \cap B_{2}\left(x_{0}\right)} K v^{p-1} \eta^{2} v^{2} \min \left\{|v|^{2 s}, L^{2}\right\} .
\end{aligned}
$$

By the Hölder and Sobolev inequalities we can write

$$
\begin{aligned}
\int_{\{v>M\} \cap B_{2}\left(x_{0}\right)} K v^{p-1} \eta^{2} v^{2} \min \left\{|v|^{2 s}, L^{2}\right\} & \leq\left(\int_{\{v>M\} \cap B_{2}\left(x_{0}\right)}\left(K v^{p-1}\right)^{N / 2}\right)^{2 / N}\|v\|_{L^{2^{*}}}^{2} \\
& \leq C\left(\int_{\{v>M\} \cap B_{2}\left(x_{0}\right)}\left(K v^{p-1}\right)^{N / 2}\right)^{2 / N} \int|\nabla v|^{2} \\
& =C \varepsilon^{-2}\left(\int_{\{v>M\} \cap B_{2}\left(x_{0}\right)}\left(K v^{p-1}\right)^{N / 2}\right)^{2 / N} \varepsilon^{2} \int|\nabla v|^{2} .
\end{aligned}
$$

If we can make $\int\left(K v^{p-1}\right)^{N / 2}$ sufficiently small, then we can bring this term on the lefthand side of $(34)$. We note that, since $\|v\|_{L^{2^{*}}\left(\mathbb{R}^{N}\right)}^{2} \leq C \varepsilon^{N}$ (by our assumptions), we have

$$
M^{2 N /(N-2)}|\{v>M\}| \leq \int v^{2^{*}} \leq C \varepsilon^{N}, \quad \text { and so } \quad|\{v>M\}| \leq C \varepsilon^{N} M^{-2 N /(N-2)} .
$$

Next, from the Hölder inequality we get

$$
\int_{\{v>M\} \cap B_{2}\left(x_{0}\right)}\left(K v^{p-1}\right)^{N / 2} \leq\left(\int_{\{v>M\}} v^{(p-1) N q / 2}\right)^{1 / q}\left(\int_{\{v>M\}} K^{N q^{\prime} / 2}\right)^{1 / q^{\prime}} .
$$

If we choose $q$ in such a way that $(p-1) N q / 2=2 N /(N-2)$, that is, if

$$
q^{\prime}=\frac{4}{4-(p-1)(N-2)},
$$

from the above estimates it follows that

$$
\begin{aligned}
& \int \varepsilon^{2}|\nabla w|^{2}+V(x) w^{2} \\
& \leq C \varepsilon^{2} \int_{B_{2}\left(x_{0}\right)} v^{2 s+2}+M^{p-1} \int_{B_{2}\left(x_{0}\right)} K \eta^{2} v^{2 s+2}+C \varepsilon^{-2}\left|x_{0}\right|^{-\beta} M^{-4 /(N-2) q^{\prime}} \varepsilon^{2} \int|\nabla v|^{2} \\
& \leq C\left(\varepsilon^{2}+M^{p-1}\left|x_{0}\right|^{-\beta}\right) \int_{B_{2}\left(x_{0}\right)} v^{2 s+2}+C \varepsilon^{-2}\left|x_{0}\right|^{-\beta} M^{-\frac{4-(p-1)(N-2)}{N-2}} \varepsilon^{2} \int|\nabla v|^{2} .
\end{aligned}
$$


Now we choose $M$ in such a way that $\varepsilon^{-2}\left|x_{0}\right|^{-\beta} M^{-\frac{4-(p-1)(N-2)}{N-2}}$ is a small constant, namely we take

$$
M=C\left(\varepsilon^{-2}\left|x_{0}\right|^{-\beta}\right)^{\frac{N-2}{4-(N-2)(p-1)}}
$$

with $C$ sufficiently large. In this way, choosing $s$ in such a way that $2 s+2=p+1$, we get

$$
\begin{aligned}
\int\left[\varepsilon^{2}|\nabla w|^{2}+V(x) w^{2}\right] & \leq C\left(\varepsilon^{2}+\left|x_{0}\right|^{-\beta} M^{p-1}\right) \int_{B_{2}\left(x_{0}\right)} v^{2 s+2} \\
& \leq C \varepsilon^{-\frac{2(p-1)(N-2)}{4-(N-2)(p-1)}} \int_{B_{2}\left(x_{0}\right)} v^{p+1} .
\end{aligned}
$$

From our assumptions on the functions $v=v_{\varepsilon}$ and the Hölder inequality it follows that

$$
\int_{B_{2}\left(x_{0}\right)} v^{p+1} \leq\left(\int_{B_{2}\left(x_{0}\right)} v^{2}\right)^{\omega}+\left(\int_{B_{2}\left(x_{0}\right)} v^{2^{*}}\right)^{1-\omega} \leq C \varepsilon^{N(1-\omega)}\left(\left|x_{0}\right|^{\alpha} \int_{B_{2}\left(x_{0}\right)} V v^{2}\right)^{\omega}
$$

for some $\omega \in(0,1)$. By Lemma 18 and the last two estimates, we obtain

$$
\begin{aligned}
& \int_{\mathbb{R}^{n}}\left[\varepsilon^{2}|\nabla w|^{2}+V(x) w^{2}\right] \\
& \leq C\left|x_{0}\right|^{d_{1}} \varepsilon^{-d_{1}} \exp \left\{-\frac{p+1}{4}\left|\log \frac{3}{4}\right| \varepsilon^{-1}\left(\left|x_{0}\right|^{(2-\alpha) / 2}-R_{\Gamma}^{(2-\alpha) / 2}\right)\right\}, \quad\left|x_{0}\right| \geq 2 R_{\Gamma}+2,
\end{aligned}
$$

for some constant $C$ depending on $\Gamma, p$ and $N$, and some positive number $d_{1}>0$, depending on $N, p, \alpha$ and $\beta$.

We note at this point that the last estimate is independent of the number $L$ in the definition of $w$. This implies that $|v|^{s+1}$ belongs to $W_{\text {loc }}^{1,2}\left(\mathbb{R}^{n}\right)$, with some quantitative estimates on the integrals, which are given in the last formula. Then the Sobolev embedding theorem implies $v \in L_{\text {loc }}^{(s+1) 2^{*}}$.

Finally, proceeding in this way and using a bootstrap argument, we obtain the result after a finite number of steps.

Remark 23. Although we already proved that $\lim _{|x| \rightarrow \infty} v_{\varepsilon}(x)=0$ for any fixed $\varepsilon>0$, the preceding lemma is needed since it gives a pointwise decay uniform in $\varepsilon$.

Lemma 24. Let $v_{\varepsilon}$ be solutions of (19) satisfying (23). Let $x_{\varepsilon}$ denote any maximum point of $v_{\varepsilon}$. Then there exists a constant $C>0, C=C(\Gamma)$, such that $\left|x_{\varepsilon}\right| \leq C$ for every $\varepsilon$ sufficiently small.

Proof. Since $x_{\varepsilon}$ is a maximum point of $v_{\varepsilon}$, one has $\Delta v_{\varepsilon}\left(x_{\varepsilon}\right) \leq 0$. Therefore, from (19) it follows that

$$
V\left(x_{\varepsilon}\right) K^{-1}\left(x_{\varepsilon}\right) \leq v_{\varepsilon}^{p-1}\left(x_{\varepsilon}\right) .
$$

From $(V)$ and $(K)$ it follows that there exists $c>0$ such that

$$
c\left|x_{\varepsilon}\right|^{\beta-\alpha} \leq V\left(x_{\varepsilon}\right) K^{-1}\left(x_{\varepsilon}\right) \text {. }
$$


From (36), 37], and (30) we deduce that if $\left|x_{\varepsilon}\right| \geq 2 R_{\Gamma}$, then

$c\left|x_{\varepsilon}\right|^{\beta-\alpha}$

$\leq\left|x_{\varepsilon}\right|^{d(p-1)} \varepsilon^{-d(p-1)} \exp \left\{-\frac{1}{4}(p-1)\left|\log \frac{3}{4}\right| \varepsilon^{-(2-\alpha) / a}\left(\left|x_{\varepsilon}\right|^{(2-\alpha) / 2}-R_{\Gamma}^{(1-\alpha) / 2}\right)\right\}$.

This immediately implies that $\left|x_{\varepsilon}\right|$ stays bounded as $\varepsilon \rightarrow 0$. Lemma24 is thereby proved.

Lemma 25. Let $v_{\varepsilon}$ be as in Lemma 24 Then there exists a constant $C>0$ such that $\left\|v_{\varepsilon}\right\|_{L^{\infty}} \geq C^{-1}$ for all $\varepsilon$ sufficiently small.

Proof. From (19) we get

$$
\left\|v_{\varepsilon}\right\|_{\varepsilon}^{2}=\int_{\mathbb{R}^{N}}\left[\varepsilon^{2}\left|\nabla v_{\varepsilon}\right|^{2}+V(x) v_{\varepsilon}^{2}\right]=\int_{\mathbb{R}^{N}} K(x) v_{\varepsilon}^{p+1} .
$$

Let us fix $\delta<\Gamma^{-(p-1) / 2}$. Then from Proposition 11 there exists $R$ such that

$$
\int_{|x|>R} K(x) v_{\varepsilon}^{p+1} d x \leq \delta \varepsilon^{-N(p-1) / 2}\left\|v_{\varepsilon}\right\|_{\varepsilon}^{p+1} .
$$

From $(V)$ and $(K)$ we have

$$
K(x) \leq \frac{k}{a} \frac{1+|x|^{\alpha}}{1+|x|^{\beta}} V(x) \leq \frac{k}{a}\left(1+R^{\alpha}\right) V(x) \quad \text { for any }|x| \leq R,
$$

hence

From this it follows that

$$
\int_{|x| \leq R} K(x) v_{\varepsilon}^{2} \leq \frac{k}{a}\left(1+R^{\alpha}\right) \int_{|x| \leq R} V(x) v_{\varepsilon}^{2} \leq \frac{k}{a}\left(1+R^{\alpha}\right)\left\|v_{\varepsilon}\right\|_{\varepsilon}^{2} .
$$

$$
\int_{|x| \leq R} K(x) v_{\varepsilon}^{p+1} \leq\left\|v_{\varepsilon}^{p-1}\right\|_{L^{\infty}} \int_{|x| \leq R} K(x) v_{\varepsilon}^{2} \leq \frac{k}{a}\left(1+R^{\alpha}\right)\left\|v_{\varepsilon}^{p-1}\right\|_{L^{\infty}}\left\|v_{\varepsilon}\right\|_{\varepsilon}^{2} .
$$

From (39), 440, and (41) we get

$$
\left\|v_{\varepsilon}\right\|_{\varepsilon}^{2}=\int_{\mathbb{R}^{N}} K(x) v_{\varepsilon}^{p+1} \leq \delta \varepsilon^{-N(p-1) / 2}\left\|v_{\varepsilon}\right\|_{\varepsilon}^{p+1}+\frac{k}{a}\left(1+R^{\alpha}\right)\left\|v_{\varepsilon}^{p-1}\right\|_{L^{\infty}}\left\|v_{\varepsilon}\right\|_{\varepsilon}^{2},
$$

which yields

$$
1 \leq \delta \varepsilon^{-N(p-1) / 2}\left\|v_{\varepsilon}\right\|_{\varepsilon}^{p-1}+\frac{k}{a}\left(1+R^{\alpha}\right)\left\|v_{\varepsilon}^{p-1}\right\|_{L^{\infty}} .
$$

Since $\left\|v_{\varepsilon}\right\|_{\varepsilon}^{p-1} \leq \Gamma^{(p-1) / 2} \varepsilon^{N(p-1) / 2}$, which follows from 23], the estimate 42 implies

$$
1 \leq \delta \widetilde{C}^{(p-1) / 2}+\frac{k}{a}\left(1+R^{\alpha}\right)\left\|v_{\varepsilon}^{p-1}\right\|_{L^{\infty}},
$$

hence, for our choice of $\delta$, we deduce that

$$
\left\|v_{\varepsilon}\right\|_{L^{\infty}}^{p-1} \geq\left(1-\delta \widetilde{C}^{(p-1) / 2}\right) \frac{a}{k\left(1+R^{\alpha}\right)}>0,
$$

which proves the lemma.

We are now in a position to characterize the ground states when $\varepsilon$ tends to 0 . 
Theorem 26. Let the assumptions of Theorem 16 hold. Then (NLS) has a (classical, positive) ground state $v_{\varepsilon}$ concentrating, as $\varepsilon \rightarrow 0$, at a global minimum point $x^{*}$ of $\mathcal{A}=V^{\theta} K^{-2 /(p-1)}$, where $\theta=(p+1) /(p-1)-N / 2$. More precisely, $v_{\varepsilon}$ has a unique maximum point $x_{\varepsilon}$ such that $x_{\varepsilon} \rightarrow x^{*}$ as $\varepsilon \rightarrow 0$, and

$$
v_{\varepsilon}(x)=U^{*}\left(\frac{x-x_{\varepsilon}}{\varepsilon}\right)+\omega_{\varepsilon}(x) \quad \text { as } \varepsilon \rightarrow 0,
$$

where $\omega_{\varepsilon} \rightarrow 0$ in $C_{\mathrm{loc}}^{2}\left(\mathbb{R}^{N}\right)$ and in $L^{\infty}\left(\mathbb{R}^{N}\right)$ as $\varepsilon \rightarrow 0$, and $U^{*}$ is the unique positive radial solution of

$$
-\Delta U^{*}+V\left(x^{*}\right) U^{*}=K\left(x^{*}\right)\left(U^{*}\right)^{p} .
$$

Proof. The proof is based upon the preceding lemmas and is rather standard (see e.g. [12, 24]). However, to keep the paper as self-contained as possible, we will carry out the arguments in detail. Let $x_{\varepsilon}$ denote a global maximum point of $v_{\varepsilon}$ (such a maximum exists since $v_{\varepsilon}(x) \rightarrow 0$ as $\left.|x| \rightarrow \infty\right)$. From Lemma 24, we know that, up to a subsequence, $x_{\varepsilon} \rightarrow x^{*}$ for some $x^{*} \in \mathbb{R}^{N}$. Set

$$
\psi_{\varepsilon}(x):=v_{\varepsilon}\left(\varepsilon x+x_{\varepsilon}\right) .
$$

Since $v_{\varepsilon}$ solves [19], $\psi_{\varepsilon}$ satisfies

$$
-\Delta \psi_{\varepsilon}(x)+V\left(\varepsilon x+x_{\varepsilon}\right) \psi_{\varepsilon}(x)=K\left(\varepsilon x+x_{\varepsilon}\right) \psi_{\varepsilon}^{p}(x), \quad x \in \mathbb{R}^{N} .
$$

From Corollary 21 and assumption $(V)$ it follows that

$$
\begin{aligned}
\Gamma & \geq \varepsilon^{-N}\left\|v_{\varepsilon}\right\|_{\varepsilon}^{2}=\varepsilon^{-N} \int_{\mathbb{R}^{N}}\left[\varepsilon^{2}\left|\nabla v_{\varepsilon}(x)\right|^{2}+V(x) v_{\varepsilon}^{2}(x)\right] d x \\
& \geq \varepsilon^{-N} \int_{\mathbb{R}^{N}}\left[\varepsilon^{2}\left|\nabla v_{\varepsilon}(x)\right|^{2}+\frac{a}{1+|x|^{\alpha}} v_{\varepsilon}^{2}(x)\right] d x \\
& =\int_{\mathbb{R}^{N}}\left[\left|\nabla \psi_{\varepsilon}(y)\right|^{2}+\frac{a}{1+\left|\varepsilon y+x_{\varepsilon}\right|^{\alpha}} \psi_{\varepsilon}^{2}(y)\right] d y .
\end{aligned}
$$

From Lemma 24 we infer that $\left|\varepsilon y+x_{\varepsilon}\right| \leq C(1+|y|)$ and therefore

$$
\int_{\mathbb{R}^{N}}\left[\left|\nabla \psi_{\varepsilon}(y)\right|^{2}+\frac{a}{1+|y|^{\alpha}} \psi_{\varepsilon}^{2}(y)\right] d y \leq C^{\prime},
$$

where $C^{\prime}$ is independent of $\varepsilon$. In particular $\left\{\psi_{\varepsilon}\right\}_{\varepsilon}$ is bounded in $C_{\text {loc }}^{\infty}$, uniformly with respect to $\varepsilon$, and we deduce that $\psi_{\varepsilon}$ converges in $C_{\text {loc }}^{2}\left(\mathbb{R}^{N}\right)$ to some $U^{*} \in C_{\text {loc }}^{2}\left(\mathbb{R}^{N}\right)$. Furthermore, using arguments similar to those carried out in the proof of Lemma 22, one infers that $\psi_{\varepsilon} \rightarrow U^{*}$ also in $L^{\infty}\left(\mathbb{R}^{N}\right)$. Passing to the limit in equation (43), we find that $U^{*} \geq 0$ is a classical solution to

$$
-\Delta U^{*}(x)+V\left(x^{*}\right) U^{*}(x)=K\left(x^{*}\right)\left(U^{*}\right)^{p}(x), \quad x \in \mathbb{R}^{N} .
$$

Moreover, since $\psi_{\varepsilon}$ attains its maximum at 0 , so does $U^{*}$. Furthermore, Lemma 25 shows that $\psi_{\varepsilon}(0)=v_{\varepsilon}\left(x_{\varepsilon}\right)=\left\|v_{\varepsilon}\right\|_{L^{\infty}} \geq C^{-1}$ for some positive constant $C$, and thus $\max U^{*}=$ $U^{*}(0) \geq C^{-1}>0$. In particular, $U^{*} \not \equiv 0$ (hence $U^{*}>0$ by the maximum principle) and is a radial function according to the Gidas-Ni-Nirenberg result [13]. Using again 
Corollary 21 we get, for any sequence $R_{n} \rightarrow \infty$,

$$
\int_{\bar{B}_{R_{n}}}\left[\left|\nabla \psi_{\varepsilon}(x)\right|^{2}+V\left(\varepsilon x+x_{\varepsilon}\right) \psi_{\varepsilon}^{2}(x)\right] d x \leq \varepsilon^{-N}\left\|v_{\varepsilon}\right\|_{\varepsilon}^{2} \leq \Gamma .
$$

Since $\psi_{\varepsilon} \rightarrow U^{*}$ in $C^{1}\left(\bar{B}_{R_{n}}\right)$, the Dominated Convergence Theorem allows us to pass to the limit in 45 , as $\varepsilon \rightarrow 0$ to obtain

$$
\int_{\bar{B}_{R_{n}}}\left[\left|\nabla U^{*}(x)\right|^{2}+V\left(x^{*}\right)\left(U^{*}\right)^{2}(x)\right] d x \leq \Gamma .
$$

Letting now $R_{n} \rightarrow \infty$, we infer that $U^{*} \in W^{1,2}\left(\mathbb{R}^{N}\right)$.

To complete the proof of Theorem 26, a further lemma is in order, which provides a lower bound for $b_{\varepsilon}$ in terms of $U^{*}$ and $x^{*}$.

Lemma 27. Let $F_{\xi}$ be as in the proof of Lemma 19 Then

$$
F_{x^{*}}\left(U^{*}\right) \leq \liminf _{\varepsilon \rightarrow 0} \varepsilon^{-N} I_{\varepsilon}\left(v_{\varepsilon}\right)=\liminf _{\varepsilon \rightarrow 0} \varepsilon^{-N} b_{\varepsilon} .
$$

Proof. One has $I_{\varepsilon}\left(v_{\varepsilon}\right)=\varepsilon^{N} \int_{\mathbb{R}^{N}} h_{\varepsilon}(x) d x$, where

$$
h_{\varepsilon}(x)=\frac{1}{2}\left|\nabla \psi_{\varepsilon}(x)\right|^{2}+\frac{1}{2} V\left(\varepsilon x+x_{\varepsilon}\right) \psi_{\varepsilon}^{2}(x)-\frac{1}{p+1} K\left(\varepsilon x+x_{\varepsilon}\right) \psi_{\varepsilon}^{p+1}(x) .
$$

Let $R>0$ to be chosen later. In view of the $C^{1}$-convergence of $\psi_{\varepsilon}$ to $U^{*}$ over the compact sets of $\mathbb{R}^{N}$ we get

$$
\begin{aligned}
& \lim _{\varepsilon \rightarrow 0} \int_{\bar{B}_{R}} h_{\varepsilon} d x \\
& \quad=\frac{1}{2} \int_{\bar{B}_{R}}\left|\nabla U^{*}\right|^{2} d x+\frac{1}{2} V\left(x^{*}\right) \int_{\bar{B}_{R}}\left(U^{*}\right)^{2} d x-\frac{1}{p+1} K\left(x^{*}\right) \int_{\bar{B}_{R}}\left(U^{*}\right)^{p+1} d x .
\end{aligned}
$$

Since $U^{*} \in W^{1,2}\left(\mathbb{R}^{N}\right)$, for any $v>0$ we can choose $R>0$ large enough such that

$$
\begin{aligned}
\lim _{\varepsilon \rightarrow 0} \int_{\bar{B}_{R}} h_{\varepsilon} d x & \geq \int_{\mathbb{R}^{N}}\left[\frac{1}{2}\left|\nabla U^{*}\right|^{2}+\frac{1}{2} V\left(x^{*}\right)\left(U^{*}\right)^{2}-\frac{1}{p+1} K\left(x^{*}\right)\left(U^{*}\right)^{p+1}\right] d x-v \\
& =F_{x^{*}}\left(U^{*}\right)-v .
\end{aligned}
$$

Let now $\eta_{R}$ be a cut-off function such that $\eta_{R}=0$ in $B_{R-1}, \eta_{R}=1$ in $\mathbb{R}^{N} \backslash B_{R}$, $0 \leq \eta_{R} \leq 1,\left|\nabla \eta_{R}\right| \leq C$, with $C$ independent of $R$. Testing (43) on $\eta_{R} \psi_{\varepsilon}$ we obtain

$$
2 \int_{\mathbb{R}^{N} \backslash B_{R}} h_{\varepsilon} d x+\left(\frac{2}{p+1}-1\right) \int_{\mathbb{R}^{N} \backslash B_{R}} K\left(\varepsilon x+x_{\varepsilon}\right) \psi_{\varepsilon}^{p+1} d x+E_{\varepsilon}=0,
$$

where

$$
E_{\varepsilon}=\int_{B_{R} \backslash B_{R-1}}\left[\nabla \psi_{\varepsilon} \cdot \nabla\left(\eta_{R} \psi_{\varepsilon}\right)+V\left(\varepsilon x+x_{\varepsilon}\right) \eta_{R} \psi_{\varepsilon}^{2}-K\left(\varepsilon x+x_{\varepsilon}\right) \eta_{R} \psi_{\varepsilon}^{p+1}\right] d x .
$$


Hence $\int_{\mathbb{R}^{N} \backslash B_{R}} h_{\varepsilon} d x \geq-E_{\varepsilon} / 2$. Again by the convergence of $\psi_{\varepsilon}$ in $C_{\text {loc }}^{1}$ to $U^{*} \in W^{1,2}\left(\mathbb{R}^{N}\right)$, we deduce that for $R$ large enough $\lim _{\varepsilon \rightarrow 0}\left|E_{\varepsilon}\right| \leq v$ and hence

$$
\liminf _{\varepsilon \rightarrow 0} \int_{\mathbb{R}^{N} \backslash B_{R}} h_{\varepsilon} d x \geq-\frac{v}{2}
$$

From (48) and 449 we conclude that

$$
\liminf _{\varepsilon \rightarrow 0} \int_{\mathbb{R}^{N}} h_{\varepsilon} d x \geq F_{x^{*}}\left(U^{*}\right)-\frac{3}{2} v
$$

for any $v>0$, and (46) follows.

Proof of Theorem 26 completed. Let us first prove that $x^{*}$ is a minimum point of the function $f(\xi)=C_{0} \mathcal{A}(\xi)$. Arguing by contradiction, we assume that there exists $\xi^{*} \in \mathbb{R}^{N}$ such that $f\left(x^{*}\right)>f\left(\xi^{*}\right)$. From (46) and (28), it follows that

$$
F_{x^{*}}\left(U^{*}\right) \leq \liminf _{\varepsilon \rightarrow 0} \varepsilon^{-N} I_{\varepsilon}\left(v_{\varepsilon}\right) \leq C_{0} \mathcal{A}(\xi), \quad \forall \xi \in \mathbb{R}^{N}
$$

On the other hand, since $U^{*}$ solves equation (44),

$$
F_{x^{*}}\left(U^{*}\right) \geq \inf _{u \in \mathcal{N}_{x^{*}}} F_{x^{*}}(u)=f\left(x^{*}\right)>f\left(\xi^{*}\right)=C_{0} \mathcal{A}\left(\xi^{*}\right)
$$

which yields a contradiction.

It remains to show that $v_{\varepsilon}$ has at most one maximum point. The proofs relies on the arguments carried out above and so we will be sketchy. By contradiction, assume that, up to a subsequence, $v_{\varepsilon}$ has two distinct maxima $x_{\varepsilon}, z_{\varepsilon}$. From Lemma 24 it follows that there exist $x^{*}, z^{*} \in \mathbb{R}^{N}$ such that $x_{\varepsilon} \rightarrow x^{*}$ and $z_{\varepsilon} \rightarrow z^{*}$. Let $\psi_{\varepsilon}$ and $U^{*}$ be as above. The convergence of $\psi_{\varepsilon}$ to $U^{*}$ in $C_{\mathrm{loc}}^{2}$ and the properties of $U^{*}$ readily imply that there exists $r>0$ such that $\psi_{\varepsilon}^{\prime \prime}(x)<$ const $<0$ for $x \in B_{r}$ provided $\varepsilon$ is small enough. Since $\varepsilon^{-1}\left(z_{\varepsilon}-x_{\varepsilon}\right)$ is a maximum point of $\psi_{\varepsilon}$, two cases can occur.

Case 1: $\varepsilon^{-1}\left(z_{\varepsilon}-x_{\varepsilon}\right)$ is bounded and hence, up to a subsequence, it converges to some $P \in \mathbb{R}^{N}$. Since $\psi_{\varepsilon}\left(\varepsilon^{-1}\left(z_{\varepsilon}-x_{\varepsilon}\right)\right)=\max \psi_{\varepsilon}$ converges to $\max U^{*}=U^{*}(0)$, we conclude that $P=0$. Therefore $\varepsilon^{-1}\left(z_{\varepsilon}-x_{\varepsilon}\right) \in B_{r}$ for $\varepsilon$ sufficiently small, which is impossible since 0 is the only critical point of $\psi_{\varepsilon}$ in $B_{r}$.

Case 2: $\varepsilon^{-1}\left(z_{\varepsilon}-x_{\varepsilon}\right)$ is unbounded, and hence it tends to $\infty$, up to a subsequence. As above, one shows that $\widetilde{\psi}_{\varepsilon} C_{\text {loc }}^{2}$-converges to $\tilde{U}^{*}$, where $\widetilde{\psi}_{\varepsilon}:=v_{\varepsilon}\left(\varepsilon x+z_{\varepsilon}\right)$ and $\tilde{U}^{*}$ is the unique positive radial solution in $W^{1,2}\left(\mathbb{R}^{N}\right)$ of

$$
-\Delta \tilde{U}^{*}(x)+V\left(z^{*}\right) \tilde{U}^{*}(x)=K\left(z^{*}\right)\left(\tilde{U}^{*}\right)^{p}(x), \quad x \in \mathbb{R}^{N} .
$$

Let us remark that, since $\left|\varepsilon^{-1}\left(z_{\varepsilon}-x_{\varepsilon}\right)\right| \rightarrow \infty$, for any $R$ the balls $\bar{B}_{R}$ and $\bar{B}^{\varepsilon}:=$ $\bar{B}_{R}\left(\varepsilon^{-1}\left(z_{\varepsilon}-x_{\varepsilon}\right)\right)$ are disjoint provided $\varepsilon$ is small enough. Using this fact and repeating 
the arguments carried out above, we readily find that for any $v>0$ it is possible to choose $R>0$ large enough such that

$$
\lim _{\varepsilon \rightarrow 0} \int_{\bar{B}^{\varepsilon}} h_{\varepsilon} \geq F_{z^{*}}\left(\tilde{U}^{*}\right)-v,
$$

as well as

$$
\liminf _{\varepsilon \rightarrow 0} \int_{\mathbb{R}^{N} \backslash\left(B_{R} \cup B^{\varepsilon}\right)} h_{\varepsilon} \geq-v .
$$

From 48, 50, and 51, we conclude that

$$
\liminf _{\varepsilon \rightarrow 0} \int_{\mathbb{R}^{N}} h_{\varepsilon} \geq F_{x^{*}}\left(U^{*}\right)+F_{z^{*}}\left(\tilde{U}^{*}\right)-3 v .
$$

Since $v$ is arbitrary we find that

$$
\liminf _{\varepsilon \rightarrow 0} \varepsilon^{-N} b_{\varepsilon} \geq F_{x^{*}}\left(U^{*}\right)+F_{z^{*}}\left(\tilde{U}^{*}\right) .
$$

From (28) and (52) it follows that $F_{x^{*}}\left(U^{*}\right)+F_{z^{*}}\left(\tilde{U}^{*}\right) \leq f\left(x^{*}\right)$. Since $x^{*}$ and $z^{*}$ are both global minimum points of $f$, we have $f\left(x^{*}\right)=f\left(z^{*}\right)$ and hence, using the definition of $f$, we deduce that

$$
F_{x^{*}}\left(U^{*}\right)+F_{z^{*}}\left(\tilde{U}^{*}\right) \leq \frac{1}{2}\left(f\left(x^{*}\right)+f\left(z^{*}\right)\right) \leq \frac{1}{2}\left(F_{x^{*}}\left(U^{*}\right)+F_{z^{*}}\left(\tilde{U}^{*}\right)\right),
$$

which is not possible. The proof is now complete.

Acknowledgements. The work has been supported by M.U.R.S.T. under the national project "Variational methods and nonlinear differential equations". Part of the work has been carried out during a visit of A.A. and A.M. at the Bernoulli Center of the EPFL, Lausanne, in the framework of the Semester "Nonlinear Analysis and Applications: Variational Methods and Nonlinear Waves". They would like to thank the Center for the kind hospitality. A.M. has also been hosted by the Mathematics Department of ETH in Zürich, to which he is very grateful.

\section{References}

[1] Ambrosetti, A., Badiale, M.: Homoclinics: Poincaré-Melnikov type results via a variational approach. Ann. Inst. H. Poincaré Anal. Non Linéaire 15, 233-252 (1998) Zbl 1004.37043 MR 99h:58135

[2] Ambrosetti, A., Badiale, M.: Variational perturbative methods and bifurcation of bound states from the essential spectrum. Proc. Roy. Soc. Edinburgh Sect. A 128, 1131-1161 (1998) Zbl 0928.34029 MR 2000a:34035

[3] Ambrosetti, A., Badiale, M., Cingolani, S.: Semiclassical states of nonlinear Schrödinger equations. Arch. Rat. Mech. Anal. 140, 285-300 (1997) Zbl 0896.35042 MR 98k:35172

[4] Ambrosetti, A., Garcia Azorero, J., Peral, I.: Remarks on a class of semilinear elliptic equations on $\mathbb{R}^{n}$, via perturbation methods. Adv. Nonlinear Stud. 1, 1-13 (2001) Zbl 1001.35038 MR 2002f:35084 
[5] Ambrosetti, A., Malchiodi, A., Ni, W.-M.: Singularly perturbed elliptic equations with symmetry: existence of solutions concentrating on spheres, Part I. Comm. Math. Phys. 235, 427466 (2003) Zbl pre01964923 MR 2004c:35014

[6] Ambrosetti, A., Malchiodi, A., Secchi, S.: Multiplicity results for some nonlinear Schrödinger equations with potentials. Arch. Rat. Mech. Anal. 159, 253-271 (2001) Zbl pre01690460 MR 2002m:35044

[7] Badiale, M., D'Aprile, T.: Concentration around a sphere for a singularly perturbed Schrödinger equation. Nonlinear Anal. 49, 947-985 (2002) Zbl 1018.35021 MR 2003f:35258

[8] Bahri, A., Li, Y. Y.: On a min-max procedure for the existence of a positive solution for certain scalar field equations in $\mathbb{R}^{N}$, Rev. Mat. Iberoamericana 6, 1-16 (1990) Zbl 0731.35036 MR 92b:35054

[9] Bahri, A., Lions P. L.: On the existence of a positive solution of semilinear elliptic equations in unbounded domains, Ann. Inst. H. Poincaré Anal. Non Linéaire 14, 365-413 (1997) Zbl 0883.35045 MR 98k:35047

[10] Berezin, F. A., Shubin, M. A.: The Schrödinger Equation. Kluwer (1991) Zbl 0749.35001

[11] Byeon, J., Wang, Z. Q.: Standing waves with a critical frequency for nonlinear Schrödinger equations, Arch. Rat. Mech. Anal. 165, 295-316 (2002) Zbl 1022.35064 MR 2003i:35250

[12] Del Pino, M., Felmer, P.: Local mountain passes for semilinear elliptic problems in unbounded domains. Calc. Var. 4, 121-137 (1996). Zbl 0844.35032 MR 97c:35057

[13] Gidas, B., Ni, W.-M., Nirenberg, L.: Symmetry of positive solutions of nonlinear elliptic equations in $\mathbb{R}^{n}$. In: Adv. Math. Suppl. Stud. Vol. 7A, A. Nachbin (ed.), 369-402 (1981) Zbl 0469.35052 MR 84a:35083

[14] Grillakis, M., Shatah, J., Strauss, W. : Stability theory of solitary waves in the presence of symmetry I. J. Funct. Anal. 74, 160-197 (1987) Zbl 0656.35122 MR 88g:35169

[15] Grossi, M.: On the number of single-peak solutions of the nonlinear Schrödinger equation. Ann. Inst. H. Poincaré Anal. Non Linéaire 19, 261-280 (2002) Zbl 1034.35127 MR 2003k:35228

[16] Li, Y. Y. : On a singularly perturbed elliptic equation. Adv. Differential Equations 2, 955-980 (1997) Zbl 1023.35500 MR 99b:35005

[17] McLeod, J. B., Stuart, C. A., Troy, W. C.: Stability of standing waves for some nonlinear Schrödinger equations. Differential Integral Equations 16, 1025-1038 (2003) Zbl pre02004939 MR 2004b:34050

[18] Noussair, E. S., Swanson, C. A.: Decaying solutions of semilinear elliptic equations in $\mathbb{R}^{N}$, SIAM J. Math. Anal. 20, 1336-1343 (1989) Zbl 0696.35051

[19] Opic, B., Kufner, A.: Hardy-type Inequalities. Pitman Res. Notes in Math. Ser. 219, Longman Sci. Tech., Harlow (1990) Zbl 0698.26007 MR92b:26028

[20] Schneider, M.: Entire solutions of semilinear elliptic problems with indefinite nonlinearities. $\mathrm{PhD}$ thesis, Univ. Mainz (2001) Zbl pre01822393

[21] Struwe, M.: Variational Methods: Applications to Nonlinear Partial Differential Equations and Hamiltonian Systems. Springer (2000) Zbl 0746.49010 MR 2000i:49001

[22] Stuart, C., An introduction to elliptic equations on $\mathbb{R}^{N}$. In: Nonlinear Functional Analysis and Applications to Differential Equations, World Sci., 237-285 (1988) Zbl 0956.35028 MR 97k:58047

[23] Stuart, C. A., Zhou, H. S.: A constrained minimization problem and its application to guided cylindrical TM-modes in an anisotropic self-focusing dielectric. Calc. Var. 16, 335-373 (2003) Zbl pre01969044 MR 2004b:49006

[24] Wang, X., Zeng, B.: On concentration of positive bound states of nonlinear Schrödinger equations with competing potential functions. SIAM J. Math. Anal. 28, 633-655 (1997) Zbl 0879.35053 MR 98e:81032 